\title{
The radio continuum perspective on cosmic-ray transport in external galaxies
}

\author{
Volker Heesen ${ }^{1}$
}

Received: 17 May 2021 / Accepted: 1 December 2021 / Published online: 14 December 2021

(c) The Author(s) 2021

\begin{abstract}
Radio continuum observations of external galaxies provide us with an excellent outside view on the distribution of cosmicray electrons in the disc and halo. In this review, we summarise the current state of what we have learned from modelling such observations with cosmic-ray transport, paying particular attention to the question to what extent we can exploit radio haloes when studying galactic winds. We have developed the user-friendly framework SPINNAKER to model radio haloes with either pure advection or diffusion, allowing us to study both diffusion coefficients and advection speeds in nearby galaxies. Using these models, we show that we can identify galaxies with winds using both morphology and radio spectral indices of radio haloes. Advective radio haloes are ubiquitous, indicating that already fairly low values of the star formation rate (SFR) surface density $\left(\Sigma_{\mathrm{SFR}}\right)$ can trigger galactic winds. The advection speeds scale with SFR, $\Sigma_{\mathrm{SFR}}$, and rotation speed as expected for stellar feedback-driven winds. Accelerating winds are in agreement with our radio spectral index data, but this is sensitive to the magnetic field parametrisation, so that constant wind speeds cannot be ruled out either. The question to what extent cosmic rays can be a driving force behind winds is still an open issue and we discuss only in passing how a simple iso-thermal wind model could fit our data. Nevertheless, the comparison with inferences from observations and theory looks promising with radio continuum offering a complementary view on galactic winds. We finish with a perspective on future observations and challenges lying ahead.
\end{abstract}

Keywords Cosmic rays · Galaxies: magnetic fields · Galaxies: fundamental parameters · Galaxies: halos · Galaxies: radio continuum

\section{Introduction}

Cosmic rays are one of the major ingredients in the interstellar medium (ISM), their energy density being comparable to that of the gaseous phases. Hence, cosmic rays play a major role in shaping the formation and evolution of galaxies in the Universe. The physics of cosmic rays is now investigated with multi-messenger astronomy (see Becker Tjus and Merten 2020, for a recent review), with a focus on the Milky Way. In recent years, nearby galaxies have become accessible both with radio continuum (Irwin et al. 2012) and $\gamma$-ray observations (Ackermann et al. 2012) to better con-

This article belongs to the Topical Collection:

Plasma, Particles, and Photons: ISM Physics Revisited.

Guest Editors: Manami Sasaki, Ralf-Jürgen Dettmar, Julia Tjus.

$凶$ V. Heesen

1 Hamburger Sternwarte, Universität Hamburg, Gojenbergsweg 112, 21029 Hamburg, Germany strain cosmic-ray transport parameters. In this review, we present some observational inferences that have been made in the past few years with improved (i.e. more sensitive) radio continuum observations, and some of the advances made modelling them. Our aims are several-fold: first, we wish to explore the physics at cloud-scale at least in an indirect way, such as the entrainment of clouds in a hot wind (Brüggen and Scannapieco 2020). Second, the global structure of the ISM dynamics is studied - something that can be well done for external galaxies - and which may inform simulations from column-type simulations that can resolve the supernova blast waves on a 10-pc scale (Girichidis et al. 2018) over global simulations of isolated galaxies (Salem and Bryan 2014; Pakmor et al. 2016a) to cosmological zoom-in simulations (Pakmor et al. 2017). Third, we can also explore the relationship with the magnetic field in the halo which fascinatingly takes the form of an X-shaped morphology (Tüllmann et al. 2000; Soida et al. 2011) and compare this with models and simulations that include the effect of magnetic fields (Pakmor et al. 2017; Steinwandel 
et al. 2020). Our work may lead eventually to the necessary understanding, so that the frequently used simple recipes for 'sub-grid physics' that are used in cosmological simulations of galaxy evolution to resemble observed galaxies (Vogelsberger et al. 2020) to put on a sound physical basis.

We will in particular address the question to what extent cosmic rays can have an influence on galaxy evolution in the form of galactic winds (see Veilleux et al. 2020, for a recent review on the cold component of winds). Cosmic rays are thought to be responsible for winds that are "cooler and smoother' (Girichidis et al. 2018) and so can lead to higher mass-loss rates than purely thermally driven winds. Also, cosmic ray-driven winds can be successful in environments that are more typical for $L_{\star}$ galaxies, such as our own Milky Way, and in particular our solar neighbourhood (Everett et al. 2008). These environments have much lower starformation rate surface densities $\left(\Sigma_{\mathrm{SFR}}\right)$ with $\Sigma_{\mathrm{SFR}} \approx 3 \times$ $10^{-3} \mathrm{M}_{\odot} \mathrm{yr}^{-1} \mathrm{kpc}^{-2}$, however, observationally the are more difficult to access than canonical star burst galaxies such as M 82 and the nuclear region in NGC 253. These 'superwind' galaxies with $\Sigma_{\mathrm{SFR}} \sim 10^{-1} \mathrm{M}_{\odot} \mathrm{yr}^{-1} \mathrm{kpc}^{-2}$ (Heckman et al. 2000) are more extreme than the relatively benign late-type galaxies that have radio haloes (Wiegert et al. 2015). Dahlem et al. (1995) already suggested a low critical $\Sigma_{\mathrm{SFR}}$-value based on radio continuum observations, which were later corroborated by optical emission line studies using integral field unit spectroscopy (Ho et al. 2016; López-Cobá et al. 2019).

More generally speaking, we can explore which effects are driving galactic winds, with processes related to stellar feedback and active galactic nuclei (AGNs) the main candidates (Yu et al. 2020). Not only the mass-loss rates, but also the composition of the wind fluid is important for galaxy evolution as is the final fate of the gas and the relation that galaxies have with the circum-galactic medium (CGM; see Tumlinson et al. 2017, for a recent review). The main questions that we would like to address with the study of radio continuum haloes (see Fig. 1) are (i) how predominant are galactic winds?; (ii) what is the role of supernovae, radiation pressure, cosmic-ray pressure, and AGN? Is there a minimum threshold of star formation or black hole activity needed to trigger cool outflows?; (iii) what is the relative distribution of the cool, warm, and hot phases in the wind? (iv) What feedback effects do they exert on the host galaxy ISM and CGM?

Cosmic rays have become recently a hot candidate to drive galactic winds, although the basic idea was already explored by Ipavich (1975). Cosmic rays have a relatively soft equation of state mean that they build up a gentle pressure gradient in the halo with a scale height of $\sim 1 \mathrm{kpc}$. This pressure gradient can gently accelerate the gas, possibly in conjunction with the hot ionised gas (Breitschwerdt et al. 1993; Everett et al. 2008; Recchia et al. 2016). In order to

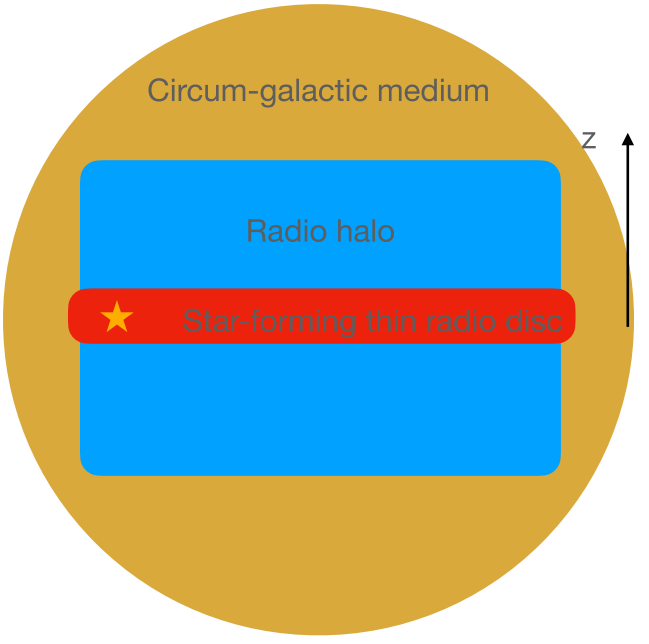

Fig. 1 The three principle components that we aim to study in the radio continuum of a galaxy as seen in the edge-on position

build up the necessary pressure gradient, cosmic rays have to first diffuse out of the star-forming regions (Salem and Bryan 2014). This can be done by either diffusion or streaming (Uhlig et al. 2012); if the cosmic rays are only passively advected, they only act as an additional pressure component and so merely puff up the gaseous disc a bit more without leading to a wind (Farber et al. 2018). Besides creating a wind, cosmic rays may play a key role in accelerating clouds of cold gas via the 'bottle neck effect' in which streaming plays an important role (Wiener et al. 2017), significantly boosting the mass-loss rate.

Radio continuum observations trace cosmic-ray electrons, the spectra of which give important clues on their transport. Early works on the integrated radio continuum spectra of galaxies showed that their curved spectra can be explained by a transition from escape-dominated radio haloes at low frequencies to radiation-loss dominated haloes at high frequencies (Pohl et al. 1991). The changing radio spectral index with distance from the star-forming midplane can be modelled with diffusion and advection, which result in different properties (Lisenfeld and Völk 2000).

The analysis of the radio spectral index in external galaxies was for a long time limited by observations, where it is relatively hard to measure the radio spectral index of extended objects using radio interferometry, for instance by the limitations due to a lack of sufficiently short base lines. However, with new instruments such as the LOw-Frequency Array (LOFAR; van Haarlem et al. 2013), the upgraded Jansky Very Large Array (JVLA; Irwin et al. 2012) and improved data reduction techniques, in particular image deconvolution with the multi-scale multi-frequency MS-MFS CLEAN algorithm (Rau and Cornwell 2011), some of these limitations have now been overcome. 


\subsection{A simplified overview of cosmic ray transport}

We follow the standard paradigm, where cosmic rays are accelerated and injected into the ISM at supernova remnants (SNRs) by diffusive shock acceleration (DSA; Bell 1978). On average, the kinetic energy per supernova is $10^{51} \mathrm{erg}$, a few per cent of which is used for the acceleration of cosmic rays (e.g. Rieger et al. 2013). The cosmic-ray luminosity of a galaxy is then (Socrates et al. 2008):

$$
L_{\mathrm{CR}}=3\left(\epsilon_{\mathrm{SN}} / 0.1\right)\left(\mathrm{SFR} / \mathrm{M}_{\odot} \mathrm{yr}^{-1}\right) \times 10^{40} \mathrm{erg} \mathrm{s}^{-1},
$$

where $\epsilon_{\mathrm{SN}}$ is the energy conversion factor from $\mathrm{SNe}$ kinetic energy into cosmic rays. Of the energy stored in the cosmic rays, between 1 and 2 per cent is channelled into the cosmicray electrons with the rest into protons and heavier nuclei (Beck and Krause 2005).

Cosmic-ray transport proceeds either by diffusion along and across magnetic field lines, cosmic-ray streaming and advection (Enßlin et al. 2011). Diffusion of cosmic rays can be understood as them being scattered at magnetic field irregularities and so following a stochastic path with a bulk speed much smaller than the speed of light. This view is corroborated by the fact that in the Milky Way the cosmic ray flux has a directional anisotropy of only $10^{-4}$ (Ahlers and Mertsch 2017). Cosmic rays reside in the Galaxy for an energy-dependent time which is $(1-2) \times 10^{7} \mathrm{yr}$ at $1 \mathrm{GeV}$ and decreases as a low fractional power of energy (Zweibel 2013). The turbulence of the magnetic fields can be either created by external processes such as supernovae and stellar winds that inject the turbulence at the tens of parsec scale, which cascades down to the cosmic-ray gyro radius; this case is usually referred to as cosmic-ray diffusion. Or cosmic rays can transfer some of their energy and momentum on the magnetic field thereby creating their own turbulence; this case is referred to as cosmic-ray streaming, where the cosmic rays follow the magnetic field lines too.

The question which values of diffusion coefficients and streaming speeds to use is of importance for numerical simulations. Values for the diffusion coefficient range from $10^{27} \mathrm{~cm}^{2} \mathrm{~s}^{-1}$ (Salem and Bryan 2014) to more conventional values of $10^{28} \mathrm{~cm}^{2} \mathrm{~s}^{-1}$ (Girichidis et al. 2018) to even larger values of $10^{29}-10^{30} \mathrm{~cm}^{2} \mathrm{~s}^{-1}$ (Hopkins et al. 2020). The canonical Milky Value of $3 \times 10^{28} \mathrm{~cm}^{2} \mathrm{~s}^{-1}$ (Strong et al. 2007) is model-dependent, particularly on the size of the halo, so that the diffusion coefficient may potentially be higher if the halo is larger. In several works, a small diffusion coefficient is argued to be of importance so that the interaction with the gas is strong enough (Pakmor et al. 2016b). In contrast, Hopkins et al. (2020) argue that the diffusion coefficient needs to be larger at $10^{29} \mathrm{~cm}^{2} \mathrm{~s}^{-1}$ so that the $\gamma$-ray flux is not too high in star-forming galaxies. If anisotropic diffusion is modelled, the ratio of perpendicular to parallel diffusion coefficients is of importance but only poorly constrained with canonical values of $D_{\perp} / D_{\|}=10-100$. Similarly, the velocity of cosmic-ray streaming is largely unknown although most theories agree that it should be of the order of the Alfvén speed. In the absence of ion-neutral damping, the wave growth of the Alfvén waves is unchecked so that cosmic rays can stream at super-Alfvénic speeds (Ruszkowski et al. 2017).

\subsection{Review structure}

A study of cosmic ray transport in external galaxies aims to determine the value of the diffusion coefficient including its energy dependence, whether diffusion proceeds isotropic or anisotropic and to what extent streaming takes over diffusion in galactic discs as the dominant transport process. In order to do this we exploit synchrotron emission from cosmic-ray electrons. As cosmic rays are injected at star formation sites, the smearing out of the radio continuum emission with respect to the star-formation distribution allows us to measure the cosmic-ray transport length. In conjunction with spectral ageing, we can model cosmic-ray transport using the electrons as proxies. This is the basic idea of our approach.

This review is structured as follows. In Sect. 2, we introduce the methodology used in order to interpret the radio continuum observations. Section 3 gives an overview of the software SPINNAKER, which we have developed to model the observations. The next three sections provide an overview on the different methods that have been used: in Sect. 4 , we present our inferences that we can gain from the vertical intensity profiles in edge-on galaxies; Sect. 5 summarises what we can learn from the radio continuum spectrum; in Sect. 6, we extend this approach to face-on galaxies. In Sect. 7, we summarise the most important results from our studies thus far. These results motivate a new approach to model radio haloes by stellar feedback-driven winds as laid out in Sect. 8. We put our results into context of inferences from absorption- and emission-line studies in Sect. 9 and to inferences from theory in Sect. 10. In Sect. 11, we discuss missing physics from our models thus far and how to address this shortcoming in the future. In Sect. 12, we summarise.

\section{Methodology}

\subsection{Radio continuum emission from galaxies}

Radio continuum emission from galaxies traces cosmic-ray electrons $\left(\mathrm{CR} e^{-}\right)$, emitting synchrotron emission while spiralling around magnetic field lines. The other contribution is from thermal emission, which stems from the free-free 
emission of thermal electrons; for this contribution, the thermal $\mathrm{H} \alpha$ emission is a good tracer and so that the emission can be separated if desired.

In the interstellar medium, $\mathrm{CR} e^{-}$are losing their energy mainly due to synchrotron and inverse Compton (IC) radiation, so that $\mathrm{GeV}$-electrons have lifetimes of a few $10^{7} \mathrm{yr}$. The ionization and bremsstrahlung losses for typical ISM densities of $n=0.05 \mathrm{~cm}^{-3}$ result in lifetimes of the order of $10^{9} \mathrm{yr}$ and can hence be neglected (Heesen et al. 2009), except at low frequencies in dense gaseous, star-forming regions (Basu et al. 2015). A comparison of $\gamma$-ray luminosity with Monte-Carlo simulations have shown that cosmic rays sample the mean density of the interstellar medium (Boettcher et al. 2013), hence such an assumption may be justified. The combined synchrotron and IC loss rate for $\mathrm{CR} e^{-}$is given by (Longair 2011):

$-\left(\frac{\mathrm{d} E}{\mathrm{~d} t}\right)=b(E)=\frac{4}{3} \sigma_{\mathrm{T}} c\left(\frac{E}{m_{\mathrm{e}} c^{2}}\right)^{2}\left(U_{\mathrm{rad}}+U_{\mathrm{B}}\right)$,

where $U_{\text {rad }}$ is the radiation energy density, $U_{\mathrm{B}}=B^{2} / 8 \pi$ is the magnetic energy density, $\sigma_{\mathrm{T}}=6.65 \times 10^{-25} \mathrm{~cm}^{2}$ is the Thomson cross-section and $m_{\mathrm{e}}=511 \mathrm{keV} \mathrm{c}^{-2}$ is the electron rest mass. The $\mathrm{CR} e^{-}$energy can be inferred from the critical frequency, where the synchrotron spectrum peaks for an individual electron (Beck 2015a):

$E \approx\left(\frac{v}{16 \mathrm{MHz}}\right)^{1 / 2}\left(\frac{\mu \mathrm{G}}{B_{\perp}}\right)^{1 / 2}$,

where $B_{\perp}$ is the total magnetic field strength perpendicular to the line of sight (i.e. in the sky plane). The time dependence of the energy for an individual $\mathrm{CR} e^{-}$is $E(t)=$ $E_{0}\left(1+t / t_{\text {syn }}\right)^{-1}$, so that at $t=t_{\text {syn }}$ the energy has dropped to half of its initial energy $E_{0}$. The $\mathrm{CR} e^{-}$synchrotron lifetime, as determined by synchrotron losses, and a smaller contribution from IC radiation losses, can be expressed by (Heesen et al. 2016):

$$
\begin{aligned}
t_{\mathrm{syn}}= & 34.2\left(\frac{v}{1 \mathrm{GHz}}\right)^{-0.5}\left(\frac{B}{10 \mu \mathrm{G}}\right)^{-1.5} \\
& \left(1+\frac{U_{\mathrm{rad}}}{U_{\mathrm{B}}}\right)^{-1} \mathrm{Myr} .
\end{aligned}
$$

If the $\mathrm{CR} e^{-}$escape time is $t_{\mathrm{esc}}$, the effective $\mathrm{CR} e^{-}$lifetime is then:

$\tau^{-1}=t_{\mathrm{syn}}^{-1}+t_{\mathrm{esc}}^{-1}$.

The $\mathrm{CR} e^{-}$injection spectrum $\mathrm{d} E N(E)=N_{0} E^{-\gamma_{\text {inj }}}$ is a power-law with an injection spectral index of $\gamma_{\text {inj }} \approx 2.2$ (fig. 3a in Caprioli 2011). Hence, the integrated radio continuum spectrum can give us important clues about the escape of $\mathrm{CR} e^{-}$because, depending on the energy dependence of the various loss processes, the injection spectrum is converted into a power-law with a different slope. For instance, the spectrum is steepened to $\propto E^{-\gamma_{\text {inj }}-1}$ if the energy losses are proportional to $E^{2}$ as is the case for both synchrotron and IC radiation losses (Longair 2011). This means that the radio spectral index is steepened to $\alpha=\alpha_{\mathrm{inj}}-0.5$, where $\alpha_{\mathrm{inj}}=\left(1-\gamma_{\mathrm{inj}}\right) / 2$ is the injection radio spectral index. ${ }^{1}$ Thus, in galaxies with free $\mathrm{CR} e^{-}$escape, the radio continuum spectrum is a power-law with $\alpha \approx-0.6$. Contrary, if the $\mathrm{CR} e^{-}$losses due to synchrotron and IC losses are important, the spectrum steepens to $\alpha \approx-1.2$ (Lisenfeld and Völk 2000).

\subsection{Advection-diffusion approximation}

The $\mathrm{CR} e^{-}$energy spectrum $N(E) \mathrm{d} E$ can be modelled by solving the diffusion-loss equation for the $\mathrm{CR} e^{-}$(e.g. Longair 2011):

$\frac{\mathrm{d} N(E)}{\mathrm{d} t}=D \nabla^{2} N(E)+\frac{\partial}{\partial E}[b(E) N(E)]+Q(E, t)$,

where $b(E)=-\mathrm{d} E / \mathrm{d} t$ for a single $\mathrm{CR} e^{-}$as given by Equation (2). Massive spiral galaxies have rather constant star formation histories, so that the $\mathrm{CR} e^{-}$injection rate can be assumed as approximately constant and so the source term $Q(E, t)$ has no explicit time dependence. If we assume that all sources of $\mathrm{CR} e^{-}$are located in the disc plane, we obtain for the source term $Q(E, t)=0$ for $z>0$ (Fig. 1). Equation (6) can be evolved in time until a stationary solution is found. We use a slightly different approach, first by restricting ourselves to a one-dimensional (1D) problem, and second by imposing a fixed inner boundary condition of $N(E, 0)=N E^{-\gamma_{\text {inj }}}$. In the stationary case, the change of the $\mathrm{CR} e^{-}$number density $\partial N / \partial t$ is solely determined by the energy loss term (second term on the right-hand side of equation (6)). Noticing that for advection we have $\partial N / \partial t=v \partial N / \partial z$, we can re-write equation (6) for the case of pure advection to:

$\frac{\partial N}{\partial z}=\frac{1}{v}\left\{\frac{\partial}{\partial E}[b(E) N(E, z)]\right\}$,

where $v$ is the advection speed, assumed here to be constant. Similarly, for diffusion we have $\partial N / \partial t=D \partial N^{2} / \partial z^{2}$ (Fick's second law of diffusion), so that we can re-write equation (6) for the case of pure diffusion to:

$\frac{\partial^{2} N}{\partial z^{2}}=\frac{1}{D}\left\{\frac{\partial}{\partial E}[b(E) N(E, z)]\right\}$,

where the diffusion coefficient can be parametrised as function of energy as $D=D_{0}(E / \mathrm{GeV})^{\mu}$. If the diffusion coefficient is energy-dependent, values for $\mu$ are thought to be

\footnotetext{
${ }^{1}$ Radio spectral indices are defined as $I_{v} \propto v^{\alpha}$.
} 
between 0.3 and 0.6 (Strong et al. 2007). For diffusion we also assume that the halo size is much larger than the $\mathrm{CR} e^{-}$ diffusion length and so the $\mathrm{CR} e^{-}$cannot escape at the halo boundary, and the decrease of the $\mathrm{CR} e^{-}$number density is solely determined by the energy losses (synchrotron and IC radiation).

If we drop the assumption of a constant advection speed, the $\mathrm{CR} e^{-}$number density will change even if the crosssectional area $A$ of the outflow is constant. According to the continuity equation:

$n_{\mathrm{CR}} v A=$ const.,

where $v$ is the advection speed and $n_{\mathrm{CR}}$ is $\mathrm{CR} e^{-}$number density. Additionally, there are adiabatic losses (cooling) that can be described as:

$-\left(\frac{\mathrm{d} E}{\mathrm{~d} t}\right)=\frac{1}{3}(\nabla \cdot v) E=\frac{E}{t_{\mathrm{ad}}}$.

For a linearly accelerating wind with a constant crosssectional area, the adiabatic loss time-scale is:

$t_{\mathrm{ad}}=3\left(\frac{\mathrm{d} v}{\mathrm{~d} z}\right)^{-1}$

An outflow that is either expanding laterally with an increasing cross-section or accelerating hence leads to adiabatic losses. Both effects can of course also work in combination, which decrease the cosmic-ray energy density, such that the cosmic rays can be in equipartition with the magnetic field. Assuming that the cosmic rays are in equipartition with the magnetic field in the disc plane, a constant advection speed in conjunction with a non-expanding outflow leads to a severe violation of equipartition in the halo (Mora-Partiarroyo et al. 2019a).

We also have to assume a magnetic field distribution. Because of simplicity we first parametrise the magnetic field as exponential distribution, so that the magnetic field strength is:

$B(z)=B_{0} \exp \left(-z / h_{\mathrm{B}}\right)$,

where $h_{\mathrm{B}}$ is the magnetic field scale height. The magnetic field strength in the mid-plane $B_{0}$ is then a fixed parameter calculated with the revised equipartition formula (Beck and Krause 2005). Alternatively, we also use a two-component exponential magnetic field:

$B(z)=B_{1} \exp \left(-z / h_{\mathrm{B} 1}\right)+B_{2} \exp \left(-z / h_{\mathrm{B} 2}\right)$,

where $h_{\mathrm{B} 1}$ and $h_{\mathrm{B} 2}$ are the magnetic field scale heights in the thin and thick radio disc, respectively, with the magnetic field strengths related as $B_{0}=B_{1}+B_{2}$. The thick radio disc is also referred to as radio halo (see Fig. 1).

\subsubsection{Cosmic-ray electron transport length}

With the most simplistic description, the cosmic-ray diffusion length can be described as:

$D=\frac{L^{2}}{4 \tau}$

where $D$ is the isotropic diffusion coefficient and $\tau$ is the $\mathrm{CR} e^{-}$lifetime. Hence, it follows that the cosmic-ray transport length $L$ scales only with the square root of the CR $e^{-}$ lifetime as $L=\sqrt{4 D \tau}$. Using convenient units, we find:

$D=0.75 \times 10^{29} \frac{(L / \mathrm{kpc})^{2}}{\tau / \mathrm{Myr}} \mathrm{cm}^{2} \mathrm{~s}^{-1}$.

Conversely, advection can be simply described as:

$v=\frac{L}{\tau}$,

where $v$ is the advection speed. Or, in convenient units:

$v=980 \frac{L / \mathrm{kpc}}{\tau / \mathrm{Myr}} \mathrm{km} \mathrm{s}^{-1}$.

For advection, the $\mathrm{CR} e^{-}$transport length scales linearly with the $\mathrm{CR} e^{-}$lifetime as $L=v \tau$. For small $\mathrm{CR} e^{-}$lifetimes, diffusion happens faster than advection and so diffusion dominates over advection near the sources in the star-forming disc. Equating diffusion and advection length, $\sqrt{4 D \tau}=v \tau$, the $\mathrm{CR} e^{-}$lifetime becomes:

$\tau=\frac{4 D}{v^{2}}$

or, in convenient units:

$\tau=12 \frac{D / 10^{28} \mathrm{~cm}^{2} \mathrm{~s}^{-1}}{\left(v / 100 \mathrm{~km} \mathrm{~s}^{-1}\right)^{2}} \mathrm{Myr}$.

Inserting this lifetime into equation (16), we obtain the cosmic-ray transport length, where the transition from diffusion to advection happens:

$z_{\star} \approx 1.2 \frac{D / 10^{28} \mathrm{~cm}^{2} \mathrm{~s}^{-1}}{v / 100 \mathrm{~km} \mathrm{~s}^{-1}} \mathrm{kpc}$.

The diffusion-dominated region near the mid-plane extends to heights of $z \lesssim z_{\star}$, whereas the advection-dominated region in the halo is at heights of $z \gtrsim z_{\star}$ (Recchia et al. 2016). In Fig. 2, we plot the $\mathrm{CR} e^{-}$number density both for advection and diffusion as function of the $\mathrm{CR} e^{-}$transport length. The transition happens at about $0.6 \mathrm{kpc}$, where for diffusion the $\mathrm{CR} e^{-}$number density drops rapidly and so advection takes over as the dominating transport mode. For the modelling of the cosmic-ray transport it is hence useful to approximate the transport by pure advection if the galaxy 


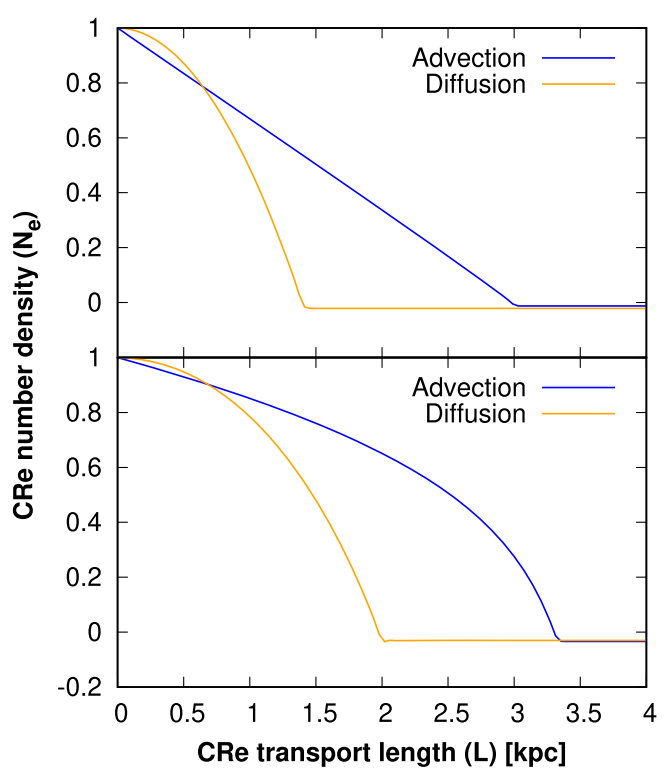

Fig. 2 Comparison of advection and diffusion, where we plot the normalised $\mathrm{CR} e^{-}$number density as function of the $\mathrm{CR} e^{-}$transport length. The $\mathrm{CR} e^{-}$injection spectral index is $\gamma_{\mathrm{inj}}=3$ (top panel) and $\gamma_{\text {inj }}=2$ (bottom panel). Further parameters as described in the text are $B=10 \mu \mathrm{G}, U_{\mathrm{rad}} / U_{\mathrm{B}}=0.3, D=10^{28} \mathrm{~cm}^{2} \mathrm{~s}^{-1}$, and $v=100 \mathrm{~km} \mathrm{~s}^{-1}$. Models adopted from Heesen et al. (2016)

has a wind because diffusion is suppressed in the halo, where we model the data. In contrast, if a galaxy has no wind, we can approximate the transport by pure diffusion. This is the approach we take in the following.

\subsection{Expected relations}

Intensity scale heights in edge-on galaxies can be used in two ways in order to investigate the cosmic-ray transport. For both methods, we use the equipartition assumption to derive the $\mathrm{CR} e^{-}$scale height from the non-thermal intensity scale height by:

$h_{e}=\frac{3-\alpha_{\mathrm{nt}}}{2} h_{\mathrm{syn}}$.

The first method is then to measure the scale height at two different frequencies (or more), where the different frequency-dependence of the scale height can be used to distinguish between advection and diffusion. Combining the $\mathrm{CR} e^{-}$synchrotron lifetime (equation (5)) with the advection transport length (equation (16)), we obtain for advection:

$h_{\mathrm{e}} \propto v^{-0.5} B^{-3 / 2}$.

Similarly, using the diffusion transport length (equation (14)), we obtain for diffusion:

$h_{\mathrm{e}} \propto v^{(\mu-1) / 4} B^{-(\mu+3) / 4}$.
Hence for diffusion, the $\mathrm{CR} e^{-}$scale height depends less on the frequency than for advection. For a possible energydependence of the diffusion coefficient, this frequency dependence of the $\mathrm{CR} e^{-}$scale height is reduced even further such that for a hypothetical, strong energy-dependence of the diffusion coefficient with $\mu=1$, the frequencydependence of the scale height even vanishes entirely.

It is important to be aware of that above relations only apply as long as the energy losses of the $\mathrm{CR} e^{-}$are high, as is for instance the case if the magnetic field strength in the halo is constant and so the $\mathrm{CR} e^{-}$lose all their energy. This scenario is referred to as the calorimetric case. More realistically, galaxies may lose some of their $\mathrm{CR} e^{-}$or the $\mathrm{CR} e^{-}$even escape almost freely from the galaxy, referred to as non-calorimetric case. For the latter, we do not expect any dependence of the scale height on frequency. This is the case if the escape time-scale:

$t_{\mathrm{esc}}=\frac{h_{\mathrm{e}}}{v}$

is much smaller than the $\mathrm{CR} e^{-}$lifetime, i.e. $t_{\mathrm{esc}} \ll \tau$.

Since the $\mathrm{CR} e^{-}$lifetime depends most on the frequency and the magnetic field strength, attempts so far have concentrated on measuring the $\mathrm{CR} e^{-}$transport length as function of them. Even more challenging is to quantify the influence of the magnetic field structure, the influence of which on the anisotropic parallel diffusion coefficient can be parametrised as (Shalchi et al. 2009):

$D_{\|} \propto\left(\frac{B_{\text {ord }}}{B_{\text {turb }}}\right)^{2} B_{\text {ord }}^{-1 / 3}$,

where $B_{\text {ord }}$ is the ordered magnetic field strength and $B_{\text {turb }}$ is the turbulent magnetic field strength. As we shall see, the main challenge is in separating the effects of spectral ageing and the influence of the magnetic field. The basic idea is to use the equations for advection and diffusion to separate them. In order to do this we implemented them in a simpleto-use computer program.

\section{An overview of SPINNAKER}

The above equations were implemented in the computer program SPectral INdex Numerical Analysis of K(c)osmicray Electron Radio-emission (SPINNAKER). ${ }^{2}$ The interactive version SPINTERACTIVE allows one the fitting of the intensities and radio spectral index profiles in a convenient way (see Fig. 3). In Table 1 we present the parameters that are fitted in each model. We now present the various options.

Before we present the various options, we briefly summarise the degeneracies involved in the empirical modelling,

${ }^{2}$ https://github.com/vheesen/Spinnaker. 


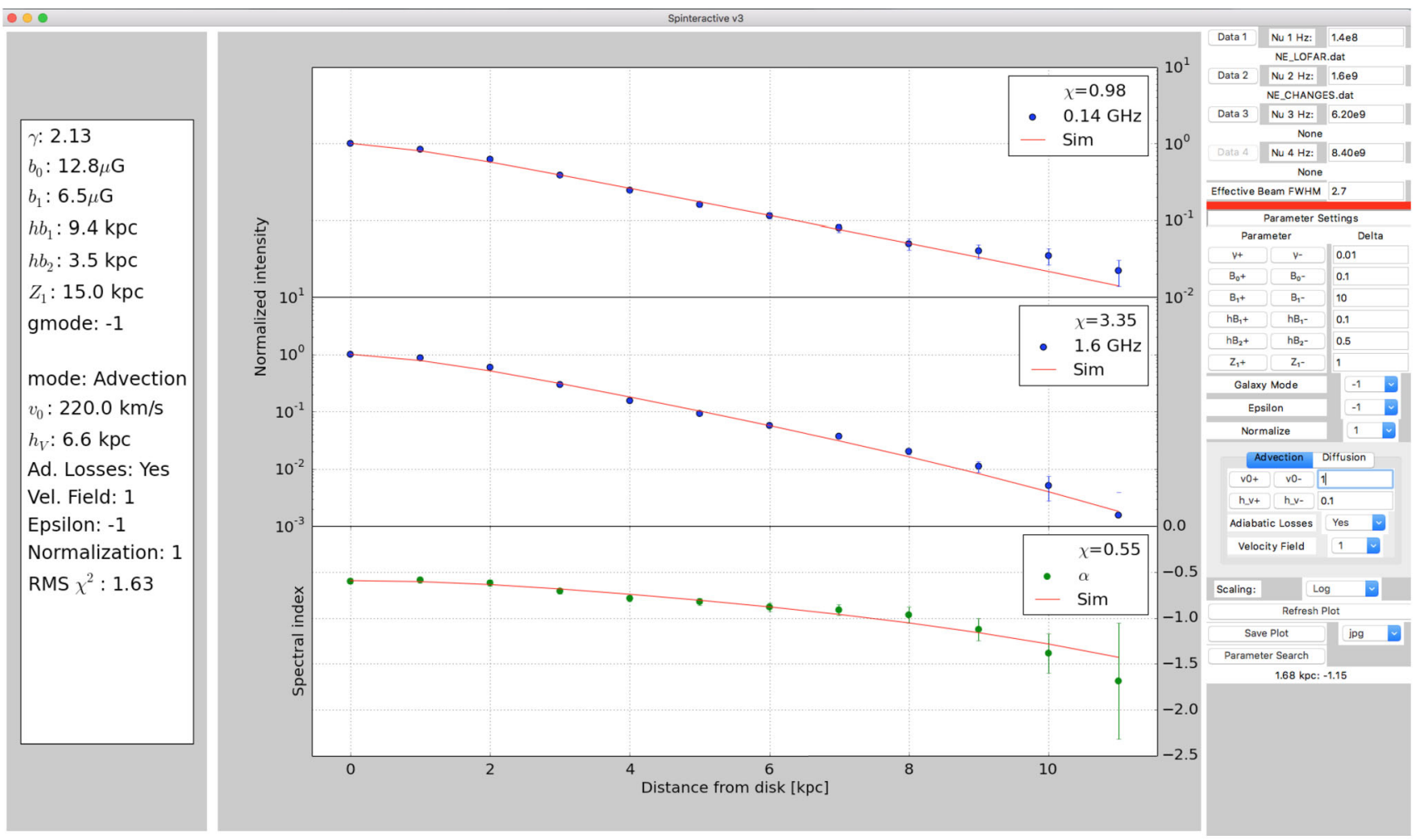

Fig. 3 SPINNAKER as viewed with SPINTERACTIVE. Application in NGC 5775 to LOFAR $150-\mathrm{MHz}$ and CHANG-ES $1.5-\mathrm{GHz}$ data. In the left panel, the parameters are summarised. The middle panel shows

in particular with respect to the $\mathrm{CR} e^{-}$density, advection velocity, and diffusion coefficient. We assume the magnetic field strength in the disc as a fixed parameter, to be measured from the energy equipartition between cosmic rays and magnetic field. The main degeneracy we have to resolve is that either a high advection speed or diffusion coefficient will lead to a higher $\mathrm{CR} e^{-}$density in the halo, which can compensate a weaker magnetic field such that it still matches the observed level of intensity. Conversely, a strong magnetic field can compensate a lower $\mathrm{CR} e^{-}$density in the halo resulting in the same radio continuum intensity. This degeneracy can be resolved by using the radio spectral index, since a higher advection speed or diffusion coefficient will lead to a flatter radio spectral index profile as the ageing of $\mathrm{CR} e^{-}$ is suppressed. This is the reason why this kind of modelling can work at all and so we get fairly reliable values for either the diffusion coefficient and/or the advection speed (Heesen et al. 2016).

\subsection{Diffusion}

Pure diffusion is chosen by mode $=1$, where the diffusion equation (8) provides us with the $\mathrm{CR} e^{-}$number density profile as presented in Fig. 4(b). As can be seen, the diffusion approximation results in flatter $\mathrm{CR} e^{-}$number density from top to bottom, the $140 \mathrm{MHz}$ data, the $1.5-\mathrm{GHz}$ data, and the radio spectral index. The right panel shows the parameters than can be interactively changed

profiles in the inner parts of the galaxy but steeper in the outskirts. The corresponding radio intensity profiles can be thus better described as Gaussian rather than as exponential functions (Heesen et al. 2019a). The models presented in Fig. 4(b) assume a non-constant, exponential magnetic field; while the magnetic field distribution influence the intensity profiles, the profiles are still markedly different from those as for advection (see also Fig. 2). We also note that the profiles of the radio spectral index are also affected by this and have a 'parabolic' shape. For diffusion we fit both the diffusion coefficient and the energy dependency $\mu$.

\subsection{Advection}

The option mode $=2$ selects pure advection for the $\mathrm{CR} e^{-}$ transport, where the $\mathrm{CR} e^{-}$number density is calculated according to equation (7).

\subsubsection{Constant advection speed}

For velocity_field $=0$, the advection speed is constant, which means the $\mathrm{CR} e^{-}$number density is regulated by radiation losses only. Hence, the $\mathrm{CR} e^{-}$number density decreases gradually with distance, different to diffusion (see 
Table 1 Parameters in SPINNAKER

\begin{tabular}{ll}
\hline Parameter & Fitted \\
\hline Magnetic field strength $B_{0}$ & fixed \\
Injection CRe spectral index $\gamma_{\text {inj }}$ & fitted \\
$\quad$ - Diffusion: mode $=1$ - & \\
Diffusion coefficient $D$ & fitted \\
Energy dependence $\mu$ & fitted \\
$\quad$ - Advection: mode $=2-$ & \\
$\quad$ Constant speed (velocity_field $=0$ ) & \\
Advection speed $v_{0}$ & \\
a Magnetic field scale height $h_{\mathrm{B}}$ & fitted \\
$\quad$ Exponential velocity profile (velocity_field $=1$ ) & fitted \\
Advection speed (at $z=0$ ) $v_{0}$ & \\
a Magnetic field scale height $h_{\mathrm{B}}$ & fitted \\
Velocity scale height $h_{v}$ & fitted \\
$\quad$ Power-law velocity profile (velocity_field $=2$ ) & fitted \\
Velocity scale height $h_{v}$ & \\
Advection speed (at $z=0)$ & fitted \\
a Magnetic field scale height $h_{\mathrm{B}}$ & fitted \\
Velocity scale height $h_{v}$ & fitted \\
Power-law index $\beta$ & fitted \\
$\quad$ Wind model (velocity_field $=3$ ) & fitted \\
Advection speed (at $z=z_{\mathrm{c}}$ ) $v_{0}$ & \\
Flux tube opening power $\beta$ & fitted \\
Flux tube scale height $z_{0}$ & fitted \\
& fitted \\
\hline &
\end{tabular}

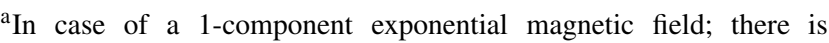
also the option to fit a 2-component exponential magnetic field (galaxy_mode $=1$ )

Fig. 4a). The radio spectral index is then also more gradually steepening in contrast to the diffusion solution, so that a linear function is a better fit.

For advection with a constant wind speed, we fit simultaneously for the advection speed $v_{0}$ and the magnetic field scale height. In principle, there is a degeneracy between the advection speed and the magnetic field scale height if only one of the intensities are studied: a smaller magnetic field scale height can be compensated by a larger advection speed. However, the radio spectral index is also very dependent on the advection speed and so a unique solution can be found (Fig. 5). Depending on whether the vertical profile needs one or two magnetic field components (equations (12) and (13)), we also may need to fit the magnetic field strength $B_{1}$ and scale height $h_{\mathrm{B} 1}$ of the thin radio disc. If the angular resolution is sufficiently high to resolve the thin disc, it may be beneficial to only fit the radio spectral index in the halo, where advection dominates (Heesen et al. 2018b).

The assumption of a constant advection speed has the advantage that the advection speeds can be accurately measured and these speeds can be regarded as a lower limits. The downside is that the cosmic-ray energy density is not in

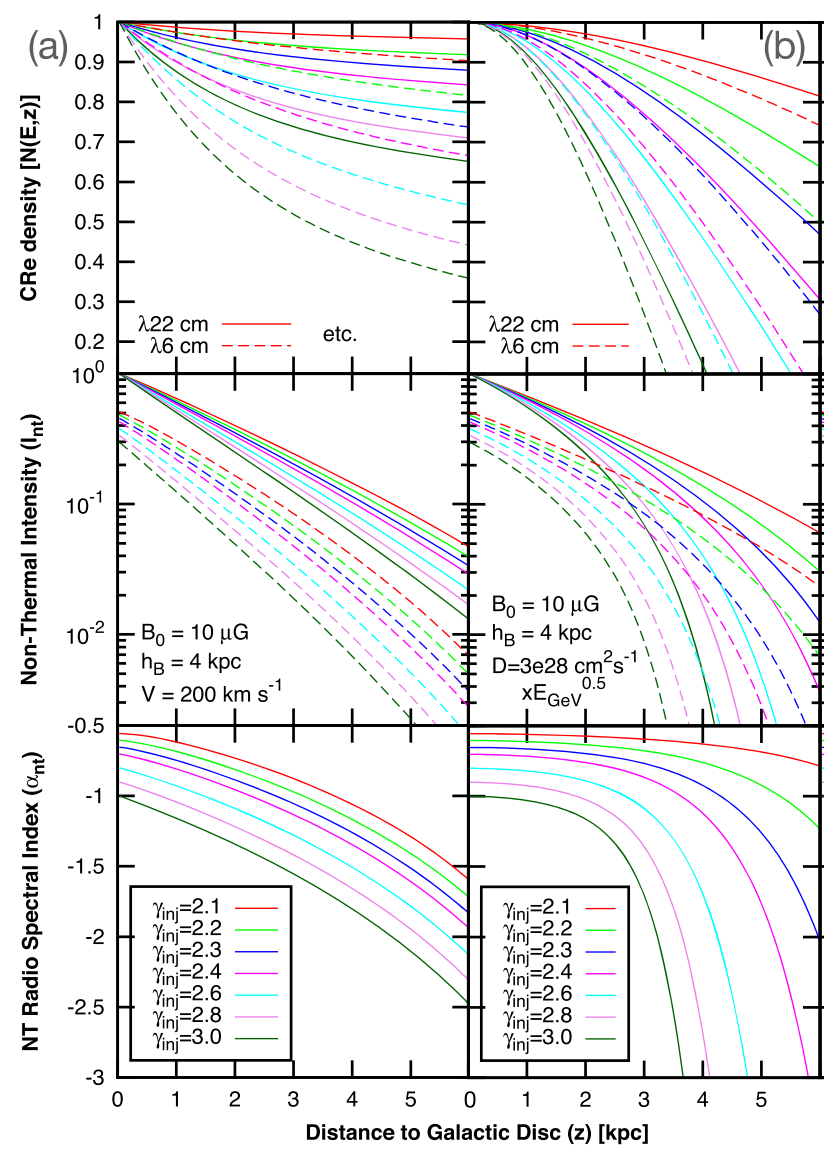

Fig. 4 Family of SPINNAKER models for various $\mathrm{CR} e^{-}$injection spectral indices at $1.4 \mathrm{GHz}$ (solid lines) and $5 \mathrm{GHz}$ (dashed lines). (a) is for advection and (b) is for diffusion. The magnetic field is a 1-component exponential function with $B=10 \mu \mathrm{G} \exp (-z / 4 \mathrm{kpc})$, the advection speed is constant with $v_{0}=200 \mathrm{~km} \mathrm{~s}^{-1}$ and the diffusion coefficient is $D=3 \times 10^{28}(E / \mathrm{GeV})^{0.5} \mathrm{~cm}^{2} \mathrm{~s}^{-1}$. The first row shows the $\mathrm{CR} e^{-}$ number density, the second row the non-thermal intensity and the third row the non-thermal radio spectral index between 1.4 and $5 \mathrm{GHz}$. From Heesen et al. (2016)

equipartition with the magnetic field for which an accelerating wind is necessary (Mora-Partiarroyo et al. 2019a).

\subsubsection{Accelerating advection speed}

If the outflow has no lateral expansion, an accelerating wind can be a way to ensure energy equipartition in the halo. We notice that radio haloes have a box-shaped outline, where the radial extent of the halo hardly changes with height and is well correlated with the size of the starforming disc (Dahlem et al. 2006; Heesen et al. 2018a; Heald et al. 2021), which argues against a strong lateral expansion. Hence, dropping the assumption of a constant advection speed, we are able to ensure energy equipartition in the halo, for instance by using an exponential velocity distribution (velocity_field $=1$ ). This introduces one more free parameter, the velocity scale height $h_{v}$, so that 


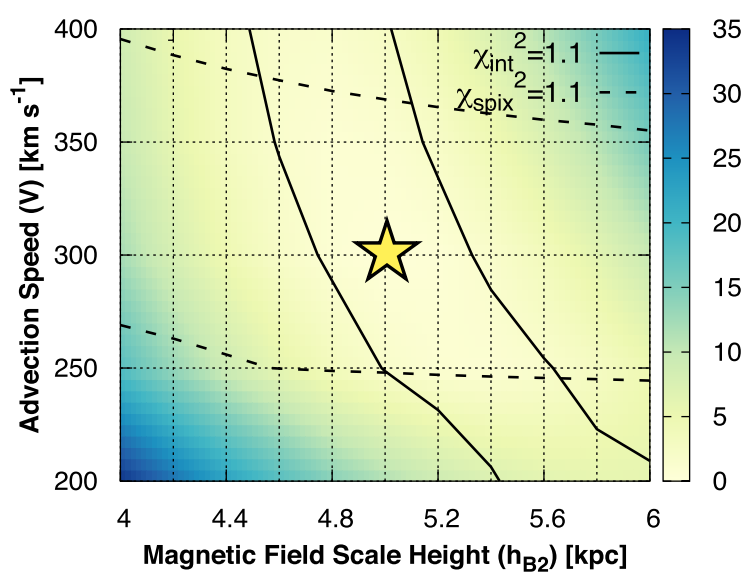

Fig. 5 Reduced $\chi^{2}$ for advection with a constant speed in the northern halo of NGC 4631. Solid lines (best-fitting intensities) define a diagonal area running from the top-left to the bottom-right, so that there is a degeneracy between advection speed and magnetic field scale height. The solution is unique though because the radio spectral index requires advection velocities as indicated by dashed lines, almost independent on the magnetic field scale height. The overlapping area in the middle with the star in the centre, define the allowed best-fitting solutions. From Heesen et al. (2018b)

the advection speed becomes $v(z)=v_{0} \exp \left(z / h_{v}\right)$. Galactic winds essentially accelerate linearly in the region where mass and energy is injected before the acceleration tailors off. This is the basic picture by the analytic wind model of Chevalier and Clegg (1985). Including different driving agents such as radiation pressure and cosmic rays change this picture only slightly (Yu et al. 2020). Heesen et al. (2018a) applied such a model successfully to the dwarf irregular galaxy IC 10. For exponential magnetic fields, energy equipartition requires $h_{v} \approx h_{B} / 2$, so that the magnetic energy density is in agreement with the cosmic-ray energy density.

Another option is a wind velocity profile with a polynominal shape (velocity_field=2), where the advection velocity is parametrised as:

$v=v_{0}\left[1+\left(\frac{z}{h_{v}}\right)^{\beta}\right]$.

For $\beta=1$, the wind is linearly accelerating, whereas for $\beta=0.5$ the wind accelerates fast in the beginning and then the acceleration tailors off. The former is a good approximation for a cosmic ray-driven wind, where both simulations (Girichidis et al. 2018) and semi-analytical 1D wind models (Everett et al. 2008) predict linear velocity profiles. The latter is a closer approximation to stellar-driven wind models (Lamers and Cassinelli 1999).

\subsubsection{Advection in a wind}

Acceleration is not the only way to achieve equipartition in the halo, the second possibility is lateral expansion. Such a geometry can be a spherical outflow, as is the case with M 82, or a flux tube geometry which has been used to model cosmic ray-driven winds. We use the latter as this better represents the morphology of radio haloes. There is a choice of magnetic field parametrisation with either a pure vertical field geometry or a helical field with both azimuthal and vertical components. Faraday rotation measurements indicate that the magnetic field in the halo may be helical (Heesen et al. 2011; Mora-Partiarroyo et al. 2019b; Stein et al. 2020), so that there is an azimuthal component as well, hence we chose such a configuration. Nevertheless, we point out that there is a degeneracy between the assumed magnetic field geometry and the acceleration of the advection speed. Changing the magnetic field strength results in different energies of the $\mathrm{CR} e^{-}$we can probe (Equation (3)), so that the spectral ageing is changed as well.

Hence, the third possibility is advection as a result of a simplified wind model using an iso-thermal wind solution (velocity_field $=3$ ). This option will be motivated in more detail in Sect. 8 (see also Heald et al. 2021). Basically, this results in an approximately linear advection speed profile with approximate energy equipartition between the cosmic rays and the magnetic field. The simplified wind equation assumes a constant sound speed (iso-thermal wind model) and a flux tube geometry (Breitschwerdt et al. 1991). This allows us to describe a stellar feedback-driven wind with few free parameters; the parameters that need to be fitted are then advection speed at the critical point $v_{0}$, the flux tube scale height $z_{0}$ and the flux tube opening parameter $\beta$. This updated model is successful in matching the vertical distribution of non-thermal radio emission, and the vertical steepening of the associated spectral index, in a consistent conceptual framework with few free parameters.

\section{Radio haloes}

Radio haloes offer us the possibility to apply the simple models of cosmic ray transport to the distribution of electrons in the halo. While some degeneracy remains between the magnetic field and the cosmic rays, the radio spectral index distribution and intensity distribution agrees to first degree with the models. This motivates to exploit the spatially resolved radio continuum emission to study cosmicray transport in more detail. Figure 6 shows two prominent radio haloes as examples of what can be seen in the radio continuum. What is immediately clear is that the morphology of the radio haloes is not like a sphere, something that has been invoked to explain the radio sky background (Singal et al. 2015). With such an outside view we can also fairly easily check the size of the radio halo, as Miskolczi et al. (2018) could show the radio halo can extend to a size of up to $10 \mathrm{kpc}$ as was also suggested by the modelling of the Milky Way halo (Orlando and Strong 2013). 


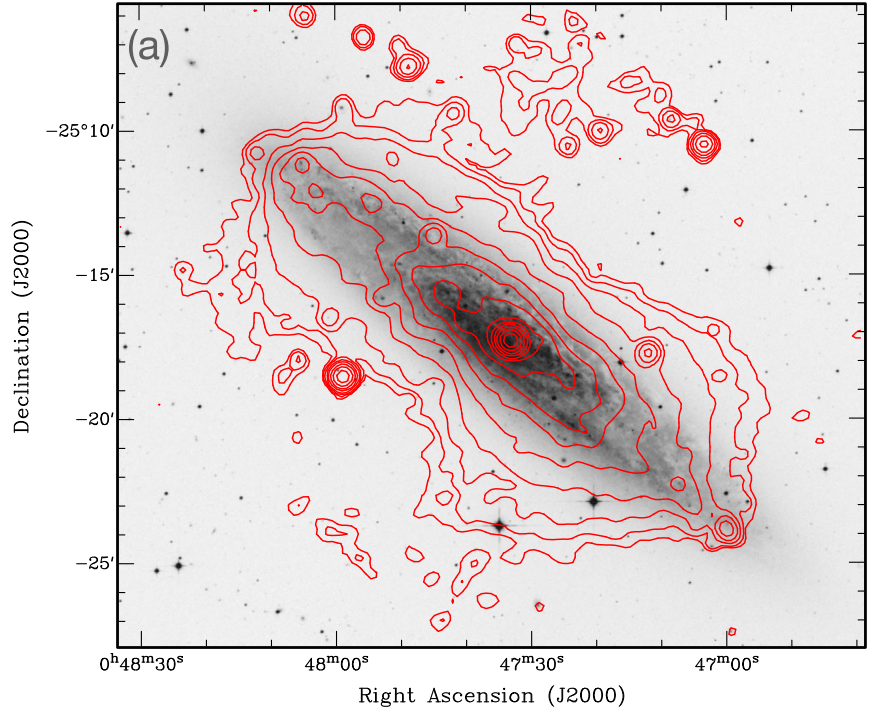

Fig. 6 Examples for two prominent dumbbell-shaped radio haloes with NGC 253 (a) and NGC 891 (b). Shown is the radio continuum intensity at $4850 \mathrm{MHz}$ (a) and $146 \mathrm{MHz}$ (b) as contours overlaid on optical im-

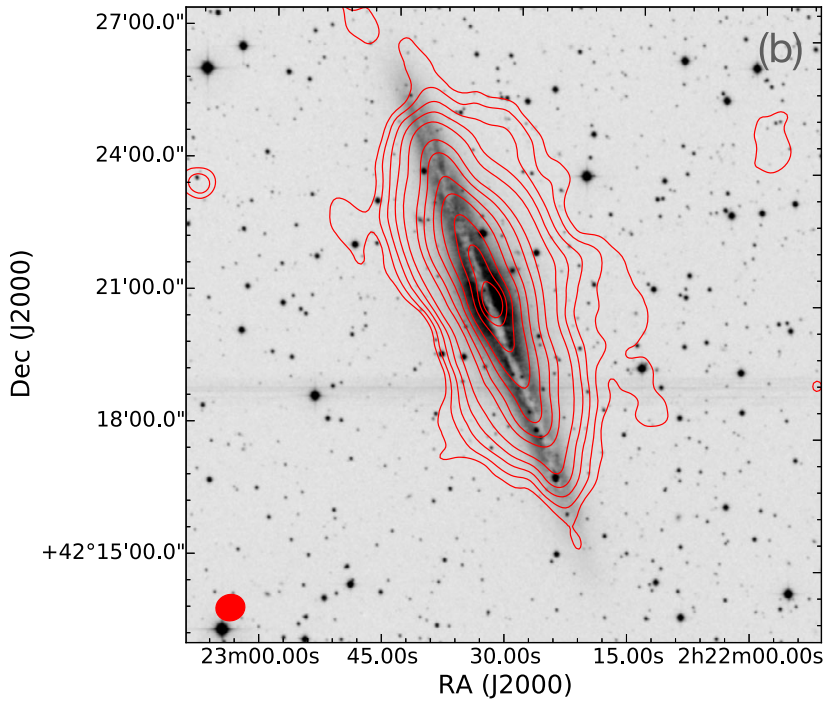

ages from the Digitized Sky Survey (DSS). From Heesen et al. (2009) (a) and Mulcahy et al. (2018) (b), respectively

Table 2 Galaxies so far analysed with SPINNAKER and re-evaluated for this review

\begin{tabular}{|c|c|c|c|c|c|c|c|c|}
\hline Galaxy & $\begin{array}{l}d^{\mathrm{a}} \\
(\mathrm{Mpc})\end{array}$ & $\begin{array}{l}\mathrm{SFR}^{\mathrm{b}} \\
\left(\mathrm{M}_{\odot} \mathrm{yr}^{-1}\right)\end{array}$ & $\begin{array}{l}\log _{10}\left(\Sigma_{\mathrm{SFR}}\right)^{\mathrm{c}} \\
\left(\mathrm{M}_{\odot} \mathrm{yr}^{-1} \mathrm{kpc}^{-2}\right)\end{array}$ & $\begin{array}{l}B_{0}{ }^{\mathrm{d}} \\
(\mu \mathrm{G})\end{array}$ & $\begin{array}{l}v_{\text {rot }}^{\mathrm{e}} \\
\left(\mathrm{km} \mathrm{s}^{-1}\right)\end{array}$ & $\operatorname{Trans}^{\mathrm{f}}$ & $\begin{array}{l}v^{\mathrm{g}} \\
\left(\mathrm{km} \mathrm{s}^{-1}\right)\end{array}$ & Reference \\
\hline IC 10 & 0.8 & 0.05 & -1.00 & 12.0 & 36 & Adv & $29 \pm 8$ & Heesen et al. (2018a) \\
\hline NGC 55 & 1.9 & 0.16 & -2.87 & 7.9 & 91 & Adv & $100 \pm 50$ & Heesen et al. (2018b) \\
\hline NGC 253 & 3.9 & 6.98 & -1.80 & 14.0 & 205 & Adv & $400 \pm 100$ & Heesen et al. (2018b) \\
\hline NGC 891 & 10.2 & 4.72 & -1.96 & 14.7 & 212 & Adv & $150 \pm 50$ & Schmidt et al. (2019) \\
\hline NGC 3044 & 4.4 & 2.03 & -2.17 & 13.1 & 153 & Adv & $200 \pm 130$ & Heesen et al. (2018b) \\
\hline NGC 3079 & 7.7 & 9.09 & -1.79 & 19.9 & 208 & Adv & $350 \pm 70$ & Heesen et al. (2018b) \\
\hline NGC 3556 & 14.09 & 4.31 & -2.26 & 9.0 & 154 & Adv & $145 \pm 30$ & Miskolczi et al. (2018) \\
\hline NGC 3628 & 14.8 & 1.73 & -2.32 & 12.6 & 215 & Adv & $250 \pm 150$ & Heesen et al. (2018b) \\
\hline NGC 4013 & 16.0 & 0.5 & -2.61 & 6.6 & 195 & Diff & $20 \pm 10$ & Stein et al. (2019b) \\
\hline NGC 4217 & 20.6 & 4.61 & -2.44 & 11.0 & 195 & Adv & $350 \pm 100$ & Stein et al. (2020) \\
\hline NGC 4565 & 11.9 & 0.73 & -2.88 & 6.0 & 244 & Diff & $60 \pm 30$ & Heesen et al. (2019b) \\
\hline NGC 4631 & 6.9 & 2.89 & -2.20 & 13.5 & 138 & Adv & $250 \pm 60$ & Heesen et al. (2018b) \\
\hline NGC 4666 & 26.6 & 16.19 & -1.58 & 18.2 & 193 & Adv & $310 \pm 50$ & Stein et al. (2019a) \\
\hline NGC 5775 & 26.9 & 9.98 & -1.81 & 16.3 & 187 & Adv & $400 \pm 80$ & Heesen et al. (2018b) \\
\hline NGC 7090 & 10.6 & 0.62 & -2.41 & 9.8 & 124 & Adv & $200 \pm 160$ & Heesen et al. (2018b) \\
\hline NGC 7462 & 13.6 & 0.28 & -2.80 & 9.7 & 112 & Diff & $90 \pm 30$ & Heesen et al. (2018b) \\
\hline
\end{tabular}

Note: It was assumed that the advection speed is constant in each of the galaxies for consistency (using velocity_field $=0$, see Sect. 3). For IC 10, NGC 891, NGC 3556, and NGC 4013 we also fitted optionally accelerating winds (see references)

${ }^{\mathrm{a}}$ Assumed distance to galaxies

${ }^{\mathrm{b}}$ Star-formation rate (SFR), calculated from either total or mid-infrared luminosity

${ }^{\mathrm{c}} \mathrm{SFR}$ surface density defined as $\Sigma_{\mathrm{SFR}}=S F R /\left(\pi r_{\star}^{2}\right)$, where $r_{\star}$ is radius of the star-forming disc

${ }^{\mathrm{d}}$ Magnetic field strength in the mid-plane as estimated with the revised equipartition formula by Beck and Krause (2005)

${ }^{\text {e}}$ Rotation speed mostly obtained from the HyperLEDA extra-galactic data base

${ }^{\mathrm{f}}$ Radio haloes with cosmic-ray transport identified either as diffusion-dominated (Diff) or advection-dominated (Adv)

gest-fitting advection speed with $1 \sigma$ uncertainties 

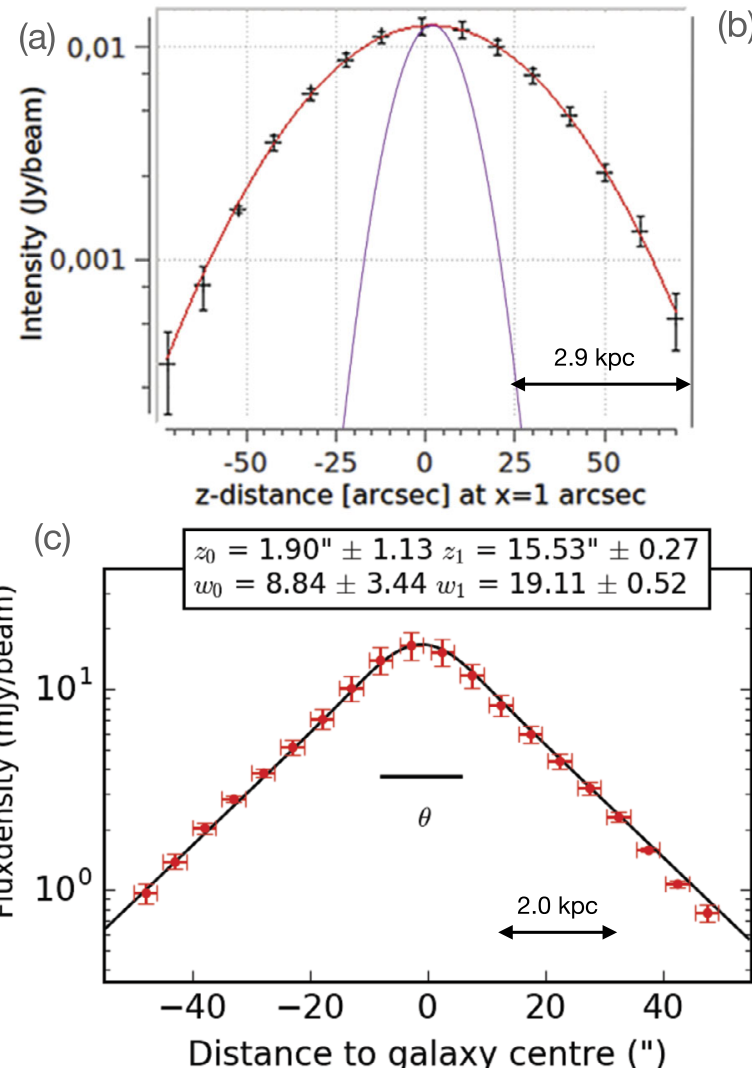

Fig. 7 Vertical intensity profiles in the haloes of four nearby edgeon spiral galaxies. The top row shows two examples for diffusiondominated radio haloes with NGC 4565 at $140 \mathrm{MHz}$ (a) and NGC 7462 at 1.4 and $4.7 \mathrm{GHz}(\mathbf{b})$. The bottom row shows two examples which are advection dominated namely NGC 4217 at $140 \mathrm{MHz}$ (c) and NGC 4631 at 1.4 and $4.9 \mathrm{GHz}$ (d). Diffusion-dominated haloes have

As this review focuses on what we have learned from the modelling with SPINNAKER, we build on the sample by Heesen et al. (2018b) who investigated 12 edge-on galaxies. Since then a few more galaxies were investigated in a similar way, so that we now have a sample of 16 galaxies that were analysed in a consistent way. In Table 2, these galaxies are listed.

\subsection{Profile shape}

Depending on the shape of the magnetic field distribution in the halo, the $\mathrm{CR} e^{-}$distribution is different for diffusion and advection, allowing us to distinguish between these two processes. Assuming an exponential magnetic field distribution is the first step since the radio continuum emission in the halo has this exponential distribution as well. Hence, the advection-diffusion approximation is used to show that diffusion leads to approximately Gaussian intensity profiles and advection leads to approximately exponential intensity profiles (see Heesen et al. 2016, and Sect. 3).
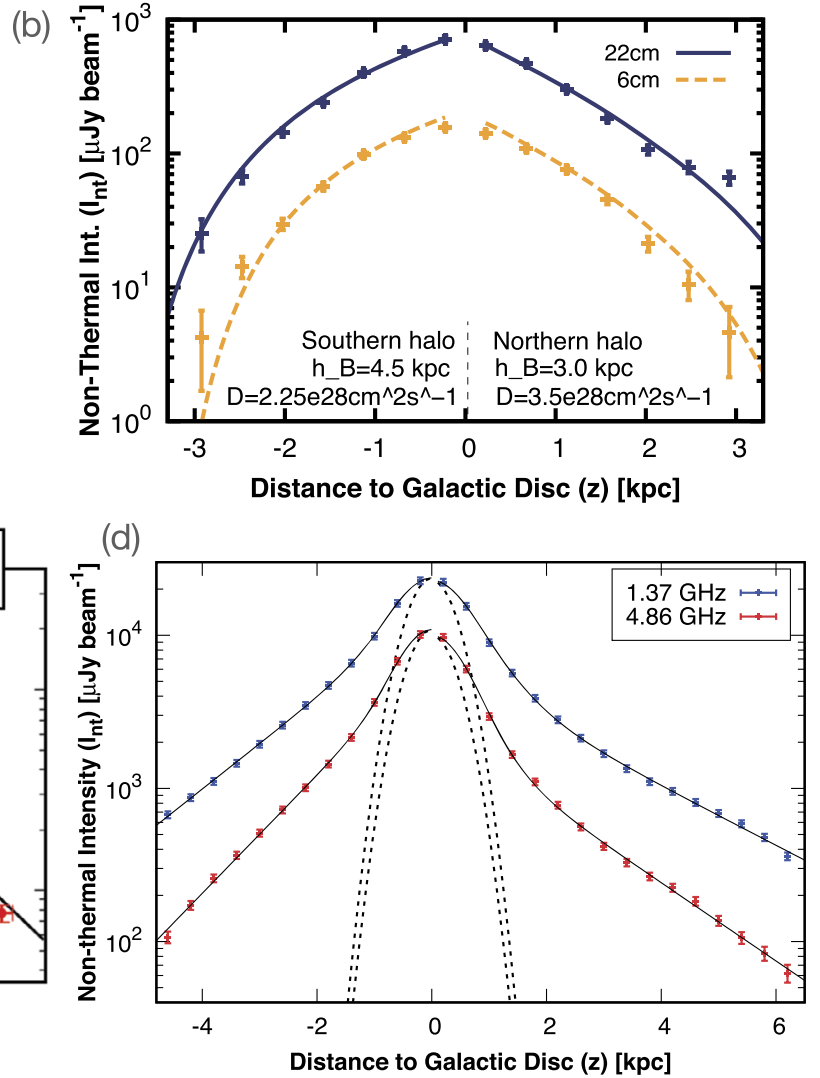

Gaussian intensity profiles (with preferentially only a 1-component profile), whereas advection-dominated radio haloes have exponential intensity profiles with preferentially 2 -component profiles. Adopted from (a) Heesen et al. (2019b), (b) Heesen et al. (2016), (c) Stein et al. (2020) and (d) Heesen et al. (2018b)

\subsubsection{Gaussian profile shape}

Examples for Gaussian radio haloes with $I_{v} \propto$ $\exp \left(-z^{2} / h_{\text {syn }}\right)$ are rare so far (see Fig. 7 (a) and (b)), with the only examples NGC 4013 (Stein et al. 2019a), NGC 4565 (Heesen et al. 2019b) and NGC 7462 (Heesen et al. 2016). What these three galaxies have in common, however, are their low star-formation rate surface densities with $\Sigma_{\mathrm{SFR}}<2 \times 10^{-3} \mathrm{M}_{\odot} \mathrm{yr}^{-1} \mathrm{kpc}^{-2}$. At these low values of $\Sigma_{\mathrm{SFR}}$, simulations suggest that the formation of outflows is suppressed (Vasiliev et al. 2019). It is an exciting prospect that radio haloes can possibly establish whether such an outflow $\Sigma_{\mathrm{SFR}}$-threshold really exists, and whether there are any other contributing factors such as a high mass-surface density.

A possible $\Sigma_{\mathrm{SFR}}$-threshold for the existence of gaseous haloes was posited already by Rossa and Dettmar (2003a), who observed the extra-planar diffuse ionised gas (eDIG) in galaxies, which was later confirmed by X-ray observation of the hot ionised gas (Tüllmann et al. 2006). These observations suggested an $\Sigma_{\mathrm{SFR}}$-threshold value similar to 
the one indicated by the diffusion-advection transition. The only other galaxy outside of this sample fitted with a single Gaussian component is NGC 4594 (M 104), an early type galaxy with a very low $\Sigma_{\text {SFR }}$ (Krause et al. 2006), hence fitting the trend.

\subsubsection{Exponential profile shape}

Most vertical intensity profiles are exponential so that $I_{v} \propto$ $\exp \left(-z / h_{\text {syn }}\right)$ with either one or two components (Krause et al. 2018), showing that they are advection dominated. If there are two components, then we refer them to a thin and thick radio disc, respectively. This is in agreement with the finding that the scale heights are almost identical at both 1.5 and $6 \mathrm{GHz}$, suggesting an almost free escape of $\mathrm{CR} e^{-}$in a wind. Of the 16 galaxies considered in this review (see Table 2), 13 have exponential radio continuum profiles (Fig. 7(c) and (d)). There are other galaxies outside of our sample, which have been fitted with exponential profiles, such as NGC 3034 (M 82; Adebahr et al. 2013).

\subsubsection{Multi-component radio disc}

It is an open question whether galaxies always have both thin and thick radio discs, as is predominantly found by observations thus far. Generally speaking, our observations thus far indicate that galaxies have either a 2-component exponential vertical distribution, consisting of both and thick radio discs, or a 1-component Gaussian disc, consisting of a thick radio disc only. Of course, this will be resolution dependent since most thin radio discs have only a scale height of a few hundred parsec (Heesen et al. 2018b), so that the angular resolution has to be sufficiently high in order to resolve them. In the sample discussed here, only 2 out of the 16 galaxies do not have a multi-component radio disc, NGC 4565 and NGC 7462, which both possess only a thick radio disc. It is notable that these two galaxies are diffusion-dominated. The only other galaxy outside of this sample that has a Gaussian vertical intensity profile is NGC 4594 (M 104), which is also fitted by a single Gaussian component (Krause et al. 2006). We can speculate that diffusion results in only a thick radio disc, whereas in the case of advection both the thin and thick discs form. Since diffusion dominates near the disc, the thin radio disc will be diffusion dominated and advection takes over as the dominating transport mode, where the profile flattens and the thick radio disc begins (Sect. 2.2.1).

Such a transition in the cosmic-ray distribution is also seen in cosmological simulations with FIRE-2, where the transition is at $10 \mathrm{kpc}$ height (which is expected as Hopkins et al. 2020, use much larger diffusion coefficients). Girichidis et al. (2018) find a flattening of the profile at $0.5 \mathrm{kpc}$ height with a more typical diffusion coefficient of $10^{28} \mathrm{~cm}^{2} \mathrm{~s}^{-1}$. As equation (20) predicts, for typical advection speeds of a few $100 \mathrm{~km} \mathrm{~s}^{-1}$ and diffusion coefficients of

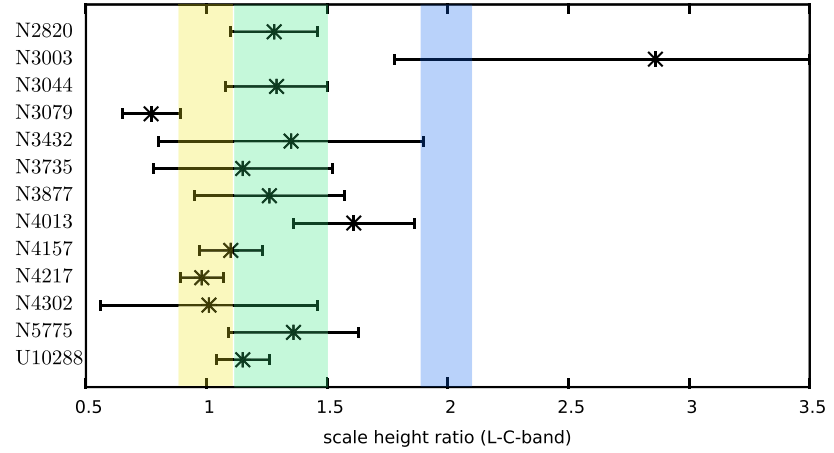

Fig. 8 The exponential radio continuum scale height in the CHANGES sample at 1.5 and $6 \mathrm{GHz}$ with the corresponding ratio. Shaded areas show the expectation for non-calorimetric advection (free escape; yellow), calorimetric diffusion (green), and calorimetric advection (blue). From Krause et al. (2018)

$10^{28} \mathrm{~cm}^{2} \mathrm{~s}^{-1}$, we expect the transition to happen at around $1 \mathrm{kpc}$ or less. Thus we raise the possibility that a galaxy with a wind has a two-component radio disc, whereas no-wind galaxies have only a one-component radio disc with a thick disc. NGC 4013 is the only galaxy that has a two-component Gaussian radio disc; this galaxy is a hybrid case where diffusion and advection both contribute because the advection speed is sufficiently slow (Stein et al. 2019b).

\subsection{Scale heights}

\subsubsection{Global measurements}

In Fig. 8, we present the scale height ratio between 1.5 and $6 \mathrm{GHz}$ in the CHANG-ES sample (Krause et al. 2018). For advection, we would expect the ratio to be 2 (equation (22)), for diffusion to be around 1.3 (depending on $\mu$; equation (23)), and for a free escape we would expect the ratio be 1 . As can be seen, the ratio is in agreement with either diffusion with a significant energy loss or free escape. What we can rule out, however, is advective transport in a calorimetric halo, although diffusive transport in a calorimetric halo would be still possible. However, there are two reasons that argue against this latter option: first, the exponential profiles are in agreement with advection; second, the galaxies have integrated radio spectral indices that are not steep enough in order classify them as $\mathrm{CR} e^{-}$calorimeters. Hence, the scale height analysis points to advective transport in winds (Krause et al. 2018).

\subsubsection{Spatially resolved measurements}

The second method using scale heights to measure $\mathrm{CR} e^{-}$ transport, is to use spatially resolved measurements. For a given galaxy, the mode of $\mathrm{CR} e^{-}$transport should not change much across the size of galaxy, for instance in a galaxywide outflow advection dominates. In this case, the local 


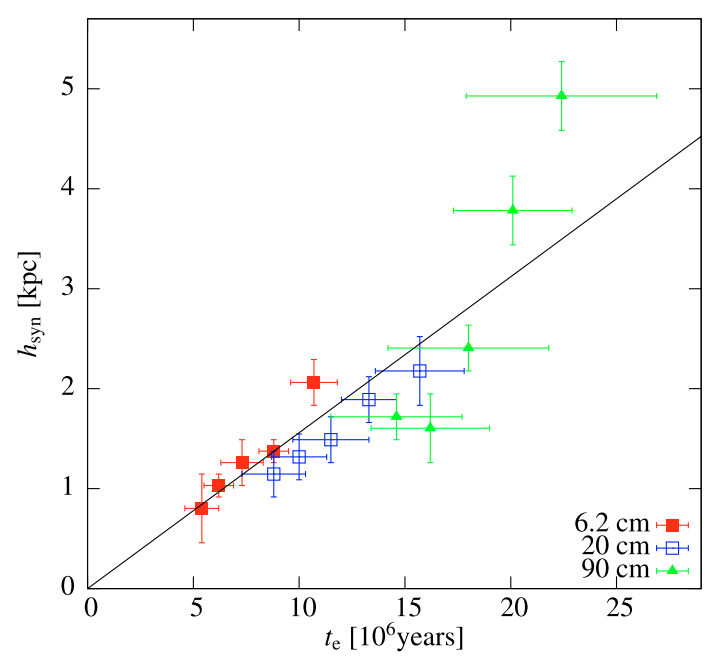

Fig. 9 The non-thermal exponential radio continuum scale height in NGC 253 as function of the $\mathrm{CR} e^{-}$synchrotron lifetime. The line shows the best-fitting advection solution. From Heesen et al. (2009)

scale height will be a function of the local $\mathrm{CR} e^{-}$lifetime, which depends on the local magnetic field strength. The motivation for this approach was the observation of radio haloes that have a 'dumbbell' shape, meaning smaller radio scale heights in the centre of the galaxy and increasing scale heights in their outskirts. Examples for this type of haloes are NGC 253 (Fig. 6(a)), NGC 891 (Fig. 6(b)) and NGC 4217 (Stein et al. 2020).

The $\mathrm{CR} e^{-}$scale height can be compared with expected relations for advection (equation (22)) and diffusion (equation (23)). The first measurement of cosmic-ray advection with this method of comparing the $\mathrm{CR} e^{-}$distribution with the magnetic field strength was presented by Heesen et al. (2009), who found that the radio continuum scale height scales linearly with the $\mathrm{CR} e^{-}$lifetime as presented in Fig. 9. Consequently, they calculated the cosmic-ray advection speed to be $v=300 \pm 30 \mathrm{~km} \mathrm{~s}^{-1}$. The alternative is to study directly the dependence of the $\mathrm{CR} e^{-}$scale height on the magnetic field strength. This has been done by Mulcahy et al. (2018) for NGC 891, who found a dependence of $h_{\mathrm{e}} \propto B^{-1.2 \pm 0.6}$, in agreement with either diffusion or advection (see Fig. 10).

\subsection{Size-scale height relation}

Krause et al. (2018) studied the scale heights in CHANGES galaxies and found that the scale height scales linearly with the size of the galaxy. In order to exclude the size of the galaxy, they defined a normalised scale height. This normalised scale height fulfils a scale height-mass surface density relation, where the normalised scale height decreases with increasing mass-surface density. Both relations point

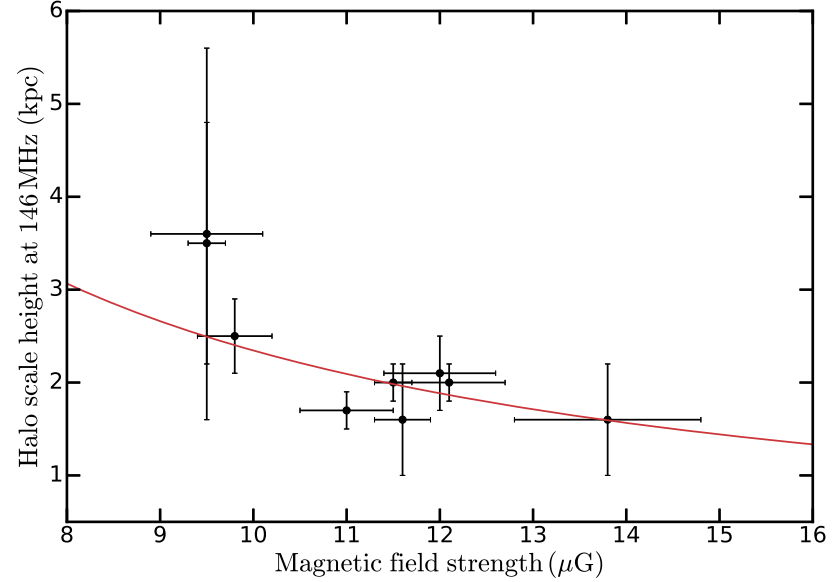

Fig. 10 The radio continuum exponential scale height at $146 \mathrm{MHz}$ in NGC 891 as function of the magnetic field strength in the mid-plane. The red line shows the best-fitting exponential function. From Mulcahy et al. (2018)

to a relation of the radio halo with stellar feedback. Interestingly, both the intensity and magnetic field scale height do not depend on either the SFR, $\Sigma_{\mathrm{SFR}}$, or rotation speed (Heesen et al. 2018b). This might point to a geometric model with an expanding outflow as well, as do the results of Krause et al. (2018).

\section{Radio continuum spectrum}

\subsection{Global spectrum}

Observations show the integrated (global) radio continuum spectrum of galaxies to be in agreement with a power-law with a non-thermal radio spectral index of -0.9 at frequencies between 1 and $10 \mathrm{GHz}$ (Tabatabaei et al. 2017). However, at low frequencies $(<1 \mathrm{GHz})$ the radio continuum spectrum deviates from a power-law and the spectrum flattens significantly (Marvil et al. 2015). The most comprehensive study to date is that of Chyży et al. (2018), who studied $\sim 100$ galaxies with LOFAR and archival data between $50 \mathrm{MHz}$ and $5 \mathrm{GHz}$. They found that the spectral index flattens by $\Delta \alpha=0.2$ from a spectral index of $\alpha=-0.77$ above $1.5 \mathrm{GHz}$ to $\alpha=-0.57$ below $1.5 \mathrm{GHz}$. Hence the low-frequency spectral index is close to the injection spectral index, which means that the $\mathrm{CR} e^{-}$may be able to escape the galaxy freely. This view is supported by the observation that the steepening of the spectrum is independent of the inclination angle (see Fig. 11). Prior, it was posed that internal effects such as due to free-free absorption at low frequencies, the spectrum is artificially flattened (Israel and Mahoney 1990). This interpretation seems to be now at least unlikely. 


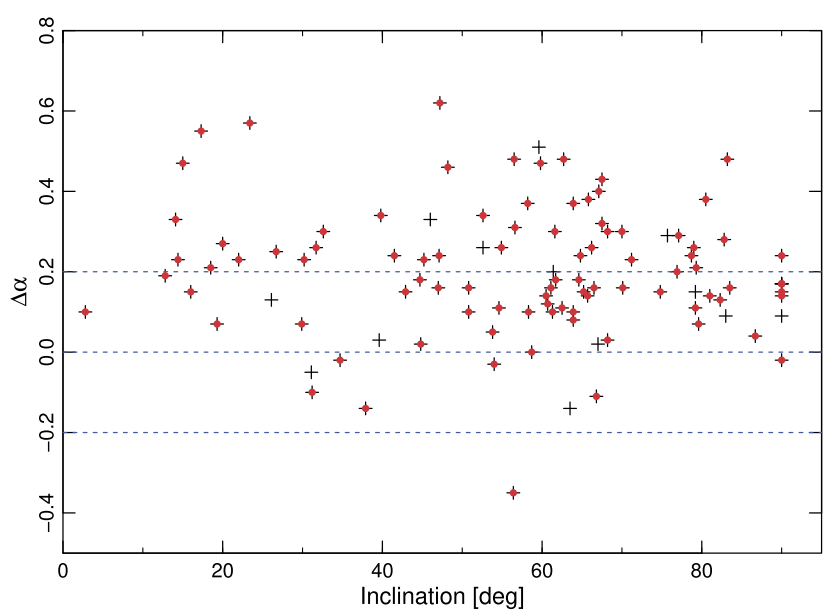

Fig. 11 Spectral curvature as function of the inclination angle. The differential spectral index is defined as $\Delta \alpha=\alpha_{\text {low }}-\alpha_{\text {high }}$, where $\alpha_{\text {low }}$ is the radio spectral index between 150 and $1500 \mathrm{MHz}$ and $\alpha_{\text {high }}$ is the radio spectral index between 1500 and $5000 \mathrm{MHz}$. From Chyży et al. (2018)

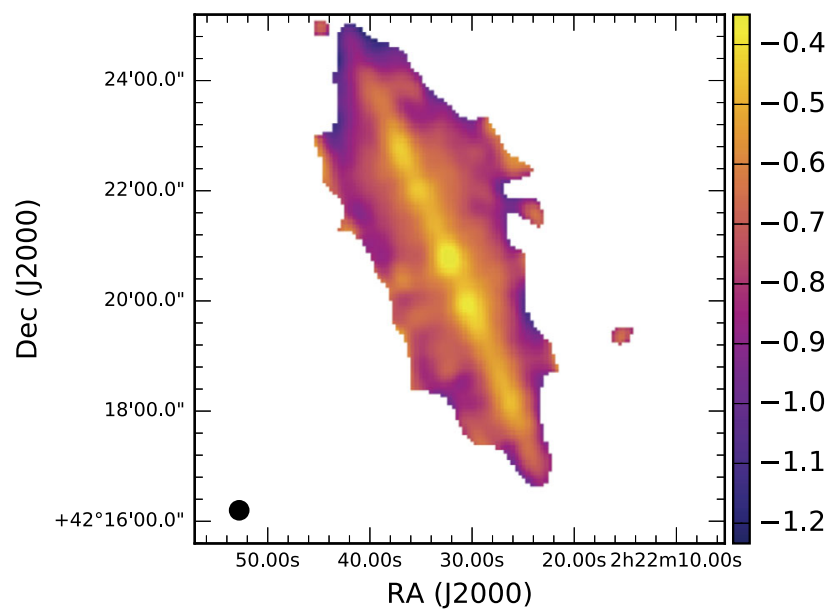

Fig. 12 The radio spectral index in NGC 891 between 146 and $1500 \mathrm{MHz}$. The spectral index is flat in the disc (although clearly nonthermal) and steepens in the halo. From Mulcahy et al. (2018)

\subsection{Spatially resolved measurements}

In edge-on galaxies, the radio spectral index can be also measured locally. Since the radio spectral index is fairly flat in the disc $(\alpha \approx-0.6)$, the star-forming galactic mid-plane, this suggests that the $\mathrm{CR} e^{-}$are freshly injected. In the halo, the radio spectral index steepens to values of $\alpha \approx-1$ or even steeper (see Fig. 12).

The next step is to use the advection-diffusion approximation to calculate vertical radio spectral index profiles. In Fig. 13, we present vertical spectral index profiles in NGC 891, which are approximately linear. The spectral index profiles show a flat spectral index in the disc, rapidly steepening in the halo, so that one finds a two-component
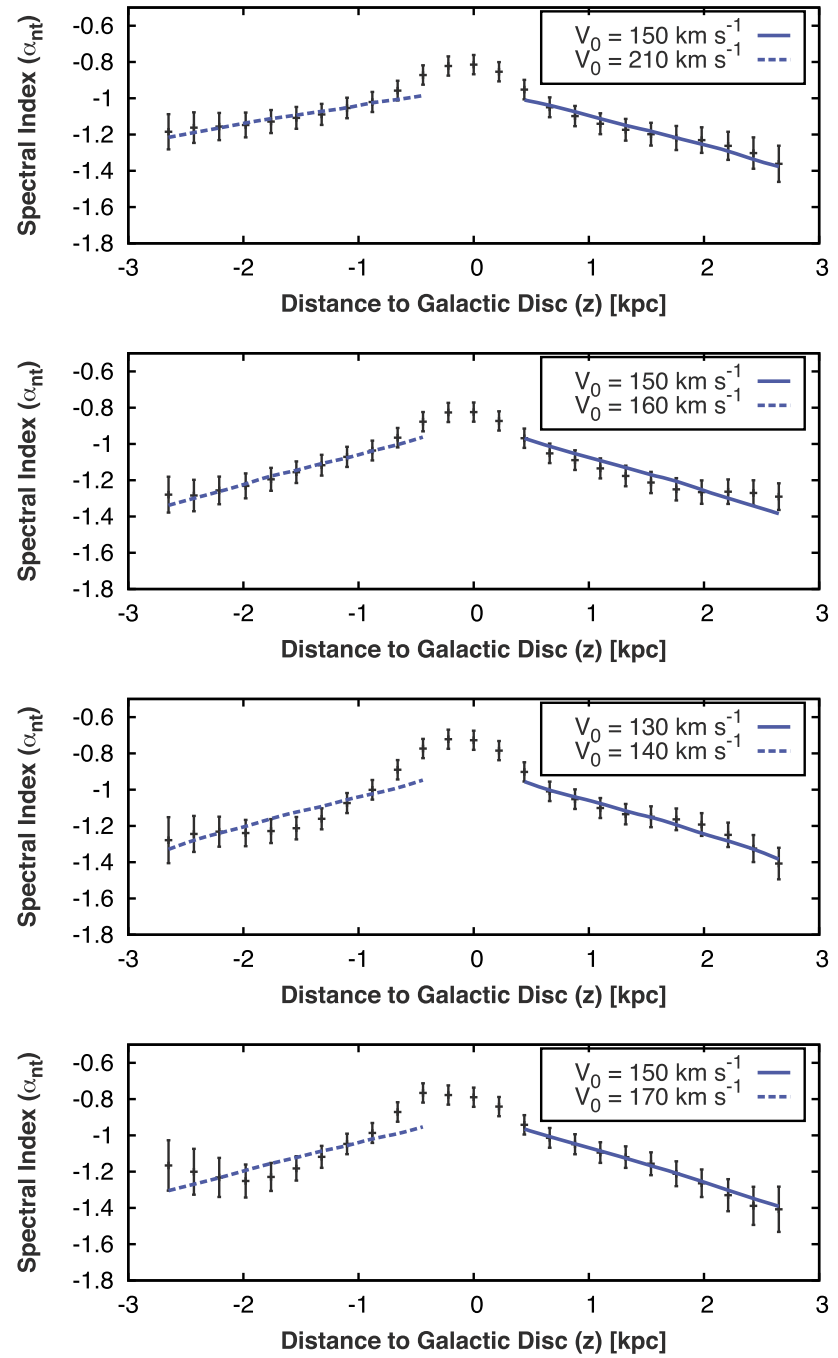

Fig. 13 Vertical profiles of the non-thermal radio spectral index between 146 and $1500 \mathrm{MHz}$ in NGC 891. Lines show best-fitting advection models, which well describe the linear decrease of the spectral index in the halo at $|z| \gtrsim 0.5 \mathrm{kpc}$. From Schmidt et al. (2019)

spectral index profile. In contrast, in Fig. 14 we present the vertical radio spectral index profile for NGC 7462, a diffusion-dominated galaxy. In this case, the spectral index is already quite steep in the disc with values of $\alpha \approx-1.2$, as would be expected for a calorimetric galaxy, with no $\mathrm{CR} e^{-}$escape. Remarkably, the radio spectral index does not steepen out to distances of $z \approx 2 \mathrm{kpc}$, quite differently to advective galaxies. The best other example for this kind of radio spectral index profiles is NGC 4565 (Schmidt et al. 2019), which has also remarkably steep spectral indices in the disc. Hence, we indeed find vertical spectral index profiles in approximate agreement with our idealised versions of the pure diffusion and advection models (Sect. 3).

This then motivates the application of the SPINNAKER models to the edge-on galaxies to decide whether they are diffusion- or advection-dominated and to measure diffusion 


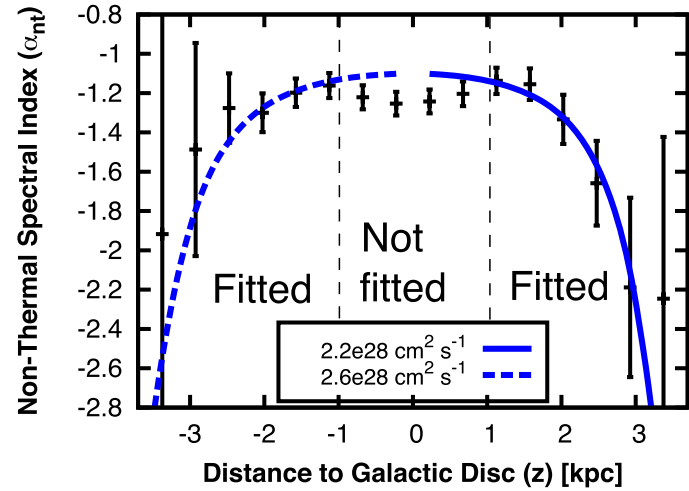

Fig. 14 Vertical radio spectral index profile between 1.4 and $4.7 \mathrm{GHz}$ in NGC 7462 as an example for diffusion-dominated transport. The profile has a 'parabolic' shape, lines show the best-fitting diffusion model, which was fitted in the halo only at $|z|>1 \mathrm{kpc}$. From Heesen et al. (2016)

coefficients and advection speeds (see Table 2). We will return to these results in Sect. 7.

\section{Face-on galaxies}

\subsection{Smoothing experiments}

A different approach of measuring the cosmic-ray transport length is to study face-on galaxies. The radio continuum emission is smoothed with respect to the star-formation rate surface density $\left(\Sigma_{\mathrm{SFR}}\right)$, which can be explained with $\mathrm{CR} e^{-}$ transport (Fig. 15). This idea was first exploited by Murphy et al. (2008) who compared the 1.4-GHz emission from the WSRT-SINGS sample by Braun et al. (2007) with 70$\mu \mathrm{m}$ far-infrared emission from Spitzer. They convolved the far-infrared map with both exponential and Gaussian kernels and minimised the difference between the convolved map and the radio continuum map. The half-length of the smoothing kernel is then the cosmic-ray transport length, which lies between 0.4 and $2.3 \mathrm{kpc}$. They found the length to be a function of the $\Sigma_{\mathrm{SFR}}$, which can be explained by increased synchrotron and IC losses and thus shorter $\mathrm{CR} e^{-}$ lifetimes.

The same approach was used in Vollmer et al. (2020), who investigated both $1.4-$ and $5-\mathrm{GHz}$ radio continuum maps. They found that the cosmic-ray transport length is a function of frequency with $1.8 \pm 0.5 \mathrm{kpc}$ at $1.4 \mathrm{GHz}$ and $0.9 \pm 0.3 \mathrm{kpc}$ at $5 \mathrm{GHz}$. They also tested both exponential and Gaussian kernels and found that the goodness of the fit cannot be used to distinguish between advection (streaming) and diffusion. However, they found that in several galaxies the 1.4/5 GHz-ratio of the transport length is larger than 1.5, an indication for streaming (equation (22)). This interpretation not dependent on the question of electron calorimetry since escape would lead to an even smaller frequency dependency. Ideally, one would like to measure the shape of the $\mathrm{CR} e^{-}$transport kernel in order to make a distinction between different models, but so far exponential and Gaussian kernels cannot be distinguished by their fitting quality alone (Murphy et al. 2008; Vollmer et al. 2020). This is easier to do in edge-on galaxies since we can measure the shape directly assuming that the $\mathrm{CR} e^{-}$are injected only in the thin star-forming disc.

\subsection{Radio-SFR relation}

A variation of the smoothing experiment (Sect. 6.1) is to study the spatially resolved radio-SFR relation, where we plot the radio continuum emission as a function of the $\Sigma_{\mathrm{SFR}}-$ values (Berkhuijsen et al. 2013). The radio continuum-starformation rate (radio-SFR) relation is approximately linear for global measurements (Heesen et al. 2014), but the spatially resolved radio-SFR relation is sub-linear with slopes of 0.6 when measured at $1-\mathrm{kpc}$ spatial resolution. The $\Sigma_{\mathrm{SFR}}{ }^{-}$ map can then be convolved with a Gaussian kernel in order to linearise the radio-SFR relation (Berkhuijsen et al. 2013; Heesen et al. 2014, 2019a). Heesen et al. (2019a) found that the half-width of the Gaussian kernel, the cosmic-ray transport length, is a function of frequency. Depending on the frequency-dependence, the transport is dominated by either by cosmic-ray diffusion or streaming.

The key finding of the spatially resolved radio-SFR relation is that the deviation from the theoretical expectation such as the Condon relation (Condon 1992) and its more recent derivatives (Murphy et al. 2011) is dependent on the radio spectral index. For a fairly flat radio spectral index of $\alpha \approx-0.6$, the deviation is small and so the relation is almost linear (red data points in Fig. 16). This fits our expectation that on a kpc-scale the radio-SFR relation is linear as long as the $\mathrm{CR} e^{-}$are young and cosmic-ray transport plays no role. Dumas et al. (2011) and Basu et al. (2015) found linear radio-SFR relations in the spiral arms of galaxies, where the spectral index is flat as well. Contrary, if the radio spectral is steep $\alpha<-0.85$, the radio continuum emission lies above the radio-SFR relation. This finding is important because it shows that spectral ageing is important shaping the relation. In areas of low star-formation rates, old $\mathrm{CR} e^{-}$have diffused into these areas and thus boost the radio continuum emission above the level of what would be expected for the local $\Sigma_{\mathrm{SFR}}$.

\subsection{Diffusion modelling}

Mulcahy et al. (2016) solved the diffusion-loss equation for radial transport of $\mathrm{CR} e^{-}$in the $1 \mathrm{D}$ case. The radial intensity profiles and spectral index profiles are fitted with the diffusion coefficient and its energy dependency. They found 

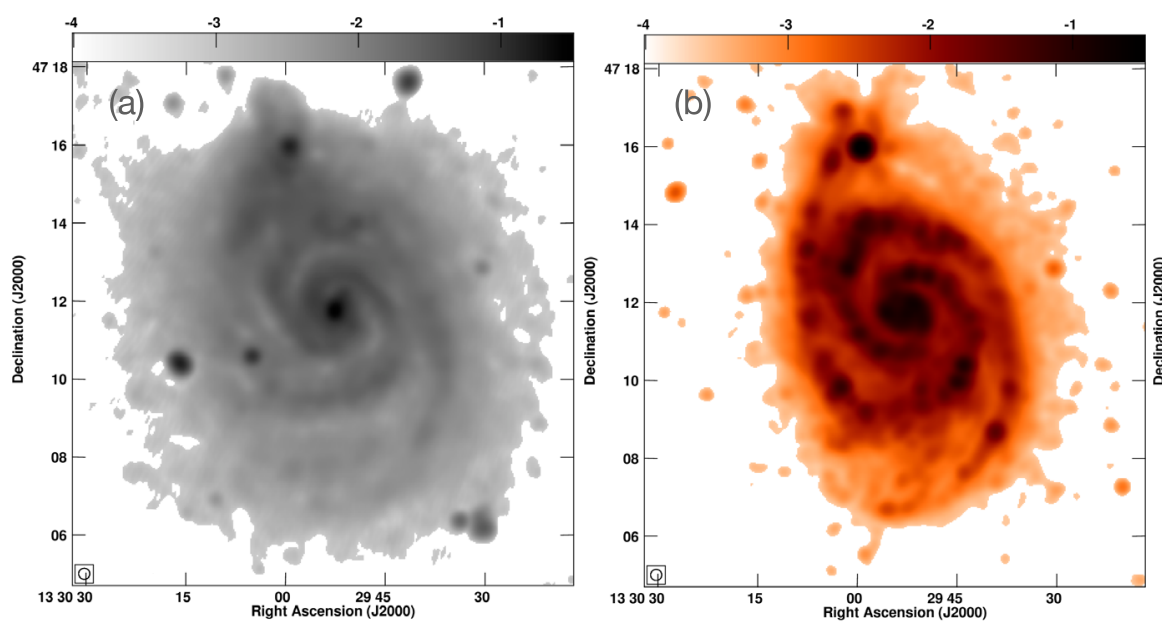

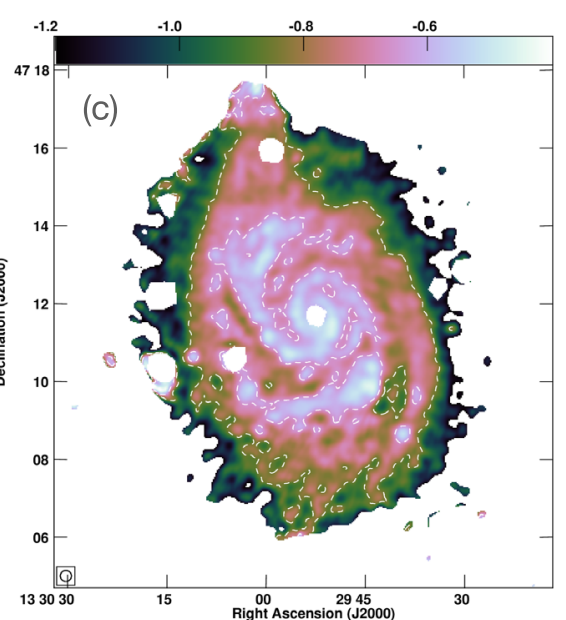

Fig. 15 Example for the influence of $\mathrm{CR}^{-}$transport in the face-on galaxy NGC 5194. The 145-MHz radio continuum map (a) is the smoothed version of the $\Sigma_{\mathrm{SFR}}$-map (b). Both maps are showing the

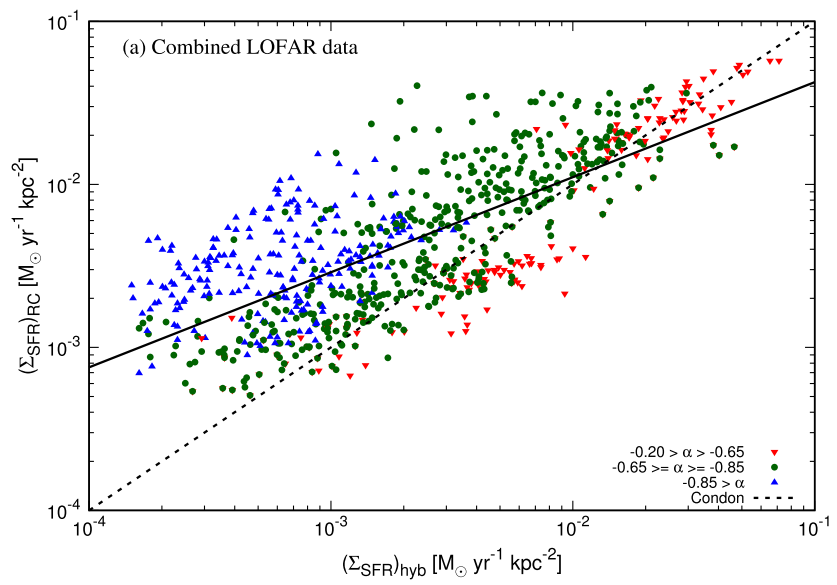

Fig. 16 Spatially resolved radio-SFR relation in four late-type galaxies at $145 \mathrm{MHz}$. The radio-derived SFR surface density is here shown as function of the mid-infrared and far-ultraviolet hybrid SFR surface density. Data points are coloured according to their radio spectral index, with red data points indicating young $\mathrm{CR} e^{-}$, green points $\mathrm{CR} e^{-}$ of intermediate age, and blue points old $\mathrm{CR} e^{-}$. The solid line shows the best-fitting relation with a sub-linear slope (when compared with the dashed 1:1 relation) that can be attributed to cosmic-ray transport. From Heesen et al. (2019a)

that a diffusion coefficient of $6 \times 10^{28} \mathrm{~cm}^{2} \mathrm{~s}^{-1}$ as their bestfitting solution. Their energy-dependence is consistent with zero, meaning that the diffusion coefficient is constant. Figure 17 shows the radial spectral index profile that they modelled. A key finding is that the spectral index is too steep without escape of $\mathrm{CR} e^{-}$. Hence, they included the diffusive escape time as:

$t_{\mathrm{esc}}=\frac{h^{2}}{D}$ star-formation rate surface density as $\log _{10}\left(\Sigma_{\mathrm{SFR}} / \mathrm{M}_{\odot} \mathrm{yr}^{-1} \mathrm{kpc}^{-2}\right)$. (c) shows the radio spectral index between 145 and $1365 \mathrm{MHz}$. From Heesen et al. (2019a)

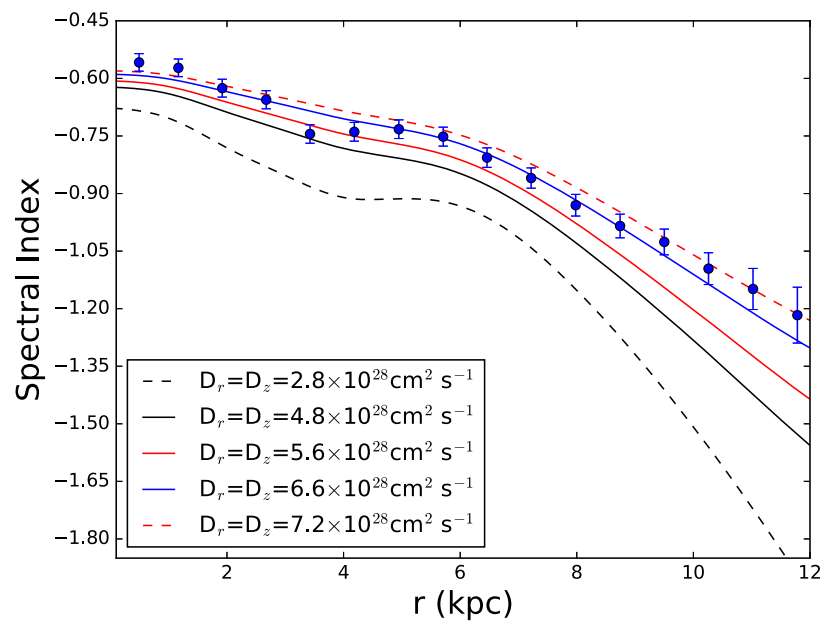

Fig. 17 The radial radio spectral index profile in NGC 5194 (M 51) between 140 and $1500 \mathrm{MHz}$. Lines show various 1D diffusion models. From Mulcahy et al. (2016)

They used H I scale heights to measure $h$, which are between 3 and $9 \mathrm{kpc}$, so that the escape time is between 11 and $88 \mathrm{Myr}$. The spectral index profile in Fig. 17 shows that the best-fitting solution. It can be seen that the model shows a smaller radial variation than the observed data, in particular the minimum at $r=4 \mathrm{kpc}$, so that a better fit might be obtained with a smaller diffusion coefficient and escape in a wind.

\section{Results}

In this section, we summarize the results that have so far been obtained for the cosmic-ray transport in external galaxies using radio continuum observations. 


\subsection{Diffusion coefficients}

The measured diffusion coefficients are between values of $10^{27}$ and $10^{29} \mathrm{~cm}^{2} \mathrm{~s}^{-1}$, with most values at around $10^{28} \mathrm{~cm}^{2} \mathrm{~s}^{-1}$ (Murphy et al. 2008, 2011; Berkhuijsen et al. 2013; Heesen et al. 2018b, 2019b; Vollmer et al. 2020). This is expected since we are tracing a few kpc-scales and the $\mathrm{CR}^{-}$lifetime is a few $10 \mathrm{Myr}$, resulting in this number using equation (15). The lowest diffusion coefficients are found in dwarf galaxies (Murphy et al. 2011; Heesen et al. 2018a) with the highest ones in radio haloes (Heesen et al. 2009, 2018b). The diffusion coefficients depend weakly on the far-infrared (SFR) surface density as Murphy et al. (2008) have shown. This is expected as long as the $\mathrm{CR} e^{-}$ lifetime is dependent on $\Sigma_{\mathrm{SFR}}$, because $t_{\text {syn }} \propto B^{-3 / 2}$ and $B \propto \Sigma_{\mathrm{SFR}}^{1 / 3}$, we expect $t_{\mathrm{syn}} \propto \Sigma_{\mathrm{SFR}}^{-1 / 2}$. Thus, the diffusion length should be $L \propto \Sigma_{\mathrm{SFR}}^{-1 / 4}$ for a non-energy dependent diffusion coefficient. This is in approximate agreement with the results of Murphy et al. (2008) although the scatter is quite significant. Tabatabaei et al. (2013) repeated this experiment and found no dependence on the SFR surface density, although their sample was fairly small.

\subsubsection{Energy dependence}

The energy dependence of the diffusion coefficient has been explored as well. There are cases when no energy dependence is needed to fit the data, such as is the case if the diffusion length scales as $L \propto t_{\mathrm{syn}}^{-1 / 2}$ or, expressed as frequency, $L \propto v^{-1 / 4}$ (Sect. 2.3). Frequently, the frequency dependence of the diffusion length $L(v)$ is flatter such as $L \propto v^{-1 / 8}$, but this can be also a result of electron non-calorimetry (Heesen et al. 2019a). The edge-on galaxy so far analysed in most detail with a pure diffusion halo, NGC 4565, is indeed better consistent with a energy independent diffusion coefficient or only a weakly dependent diffusion coefficient (Heesen et al. 2019b; Schmidt et al. 2019). Essentially, an energydependent diffusion coefficient would lead to an even more pronounced curvature of the radio spectral index profile than what is observed. Heesen et al. (2016) tested the energydependence in NGC 7462, but did not find a strong indication for it.

A different approach is to fit the Gaussian convolution kernel in face-on galaxies with a cosmic-ray diffusion model. Heesen et al. (2019a) did this and found the energy dependence to vary widely with $\mu=0-0.6$. There are indications, however, in particular from the radio spectral index, that high values of $\mu$ are the result of $\mathrm{CR} e^{-}$escape (electron non-calorimetry) rather than an intrinsic feature of the $\mathrm{CR} e^{-}$transport. In summary, diffusion coefficients in the $\mathrm{GeV}$-range seem to be not energy dependent. In those cases where we see a dependence, the indication is either weak (in edge-on galaxies) or can be largely explained by flat radio spectral indices hinting at $\mathrm{CR} e^{-}$escape (in face-on galaxies). The observation that for a few $\mathrm{GeV}$ the diffusion coefficient is not energy-dependent is in agreement with the Boron-to-Carbon (secondary to primary) cosmic-ray ratio in the Milky Way (Becker Tjus and Merten 2020).

\subsection{Cosmic-ray streaming}

The indications for cosmic-ray streaming come mostly from scaling of the $\mathrm{CR} e^{-}$transport length with frequency, which in case of streaming resembles advection rather than diffusion (Sect. 2.3). Vollmer et al. (2020) found two galaxies where the $\mathrm{CR} e^{-}$transport length scales more with the frequency than can be explained by pure diffusion even when the diffusion coefficient is assumed to be energy independent. Similarly, Beck (2015b) found in IC 342 the CR $e^{-}$ propagation length to scale with $L \propto v^{-0.5}$. This can be explained by cosmic-ray streaming, where the $\mathrm{CR} e^{-}$are transported with a constant speed, for instance the Alfvén speed. We may consider the influence of an advection-dominated radio halo, which would result in a similar behaviour. What argues against such a halo is that an advective halo will limit the confinement of cosmic rays, which would again limit the effective $\mathrm{CR} e^{-}$lifetime and thus reduce the frequency dependence. Taken together, the results by Beck (2015b) and Vollmer et al. (2020) seem to be strongly indicative of cosmic-ray streaming. Another hint comes from Tabatabaei et al. (2013) who found that the $\mathrm{CR} e^{-}$transport length in NGC 6946 is larger than what one would expect from the ratio of ordered and turbulent magnetic field strength.

In edge-on galaxies, cosmic rays can stream from the disc into the halo along vertical magnetic field lines. Obviously, in diffusion-dominated galaxies streaming must be suppressed, so that we can assume that galaxies without outflows do not have the right type of magnetic field structure, presumably lacking vertical magnetic field lines. Indeed, the two pure diffusion haloes in our sample, NGC 4565 and NGC 7462, have no dominant vertical magnetic field lines (Heesen et al. 2016; Wiegert et al. 2015). The hybrid diffusion-advection galaxy NGC 4013 has at least a significant vertical magnetic field component (Stein et al. 2019a). In galaxies with winds, advection and streaming may be observed together although a separation of them is difficult. In the edge-on galaxy NGC 5775, the vertical radio spectral index gradient is much reduced at the position of vertical magnetic field lines (Duric et al. 1998; Heald et al. 2021). This could also be the result of $\mathrm{CR} e^{-}$streaming; the effective $\mathrm{CR} e^{-}$bulk speed is then the superposition of Alfvén and wind speed.

\subsection{Anisotropic diffusion}

The question whether diffusion happens isotropic or anisotropic is of importance for the modelling of galaxy evolution. 

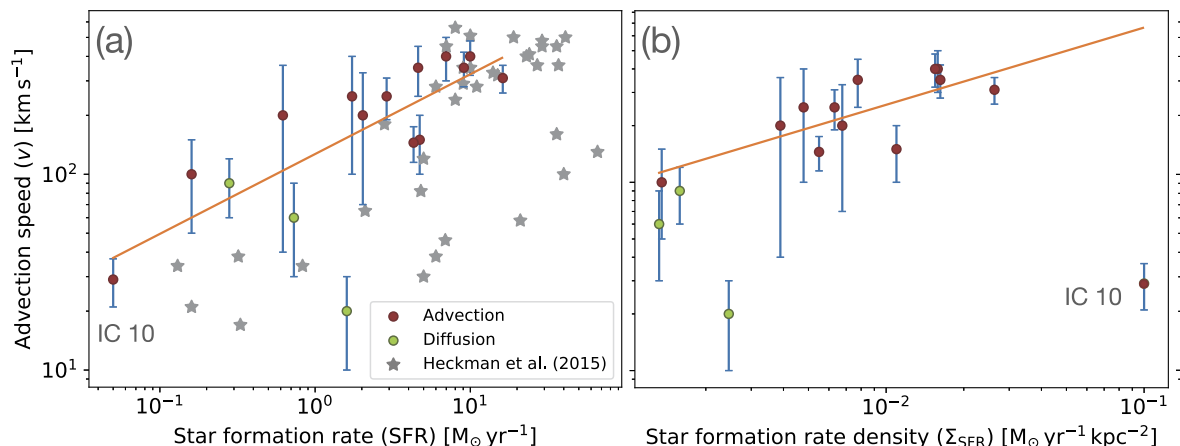

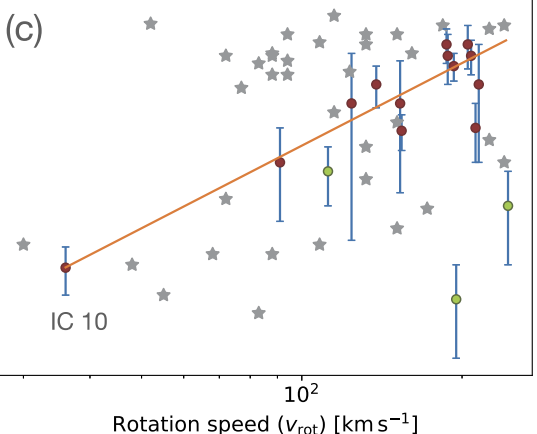

Fig. 18 Re-evaluated scaling relations of the $\mathrm{CR} e^{-}$advection speed in edge-on galaxies. (a) shows the advection speed as function of SFR, (b) as function of SFR surface density and (c) as function of the rotation speed. Best-fitting advection scaling relations (Table 3) are shown as solid lines. Values for the filled circular data points are from Table 2; grey stars show UV absorption line measurements for a different sample of galaxies which were taken from Heckman et al. (2015)

parallel diffusion coefficient, which involves the turbulent component of the magnetic field which can be described by the so-called correlation length (similar to the field line bend-over length).

\subsection{Advection speed scaling relations}

The advection speed scaling relations with SFR, $\Sigma_{\mathrm{SFR}}$, and the rotation speed $v_{\text {rot }}$ were already investigated by Heesen et al. (2018b). For this review, we have re-evaluated their sample which we extended to 16 galaxies (Table 2). In our sample, three galaxies are diffusion-dominated, which we exclude in the fitting process but present them in the plots for comparison. In Table 3 an overview of the scaling relations discussed can be found.

The advection speed as function of the SFR is presented in Fig. 18(a), where the advection speed scales with the SFR as $v \propto S F R^{0.4}$. Similarly, the advection speed scales with the SFR surface density as $v \propto \Sigma_{\mathrm{SFR}}^{0.4}$ as shown in Fig. 18(b). However, this relation only holds if the starburst dwarf irregular galaxy IC 10, analysed by Heesen et al. (2018a), is excluded from the fitting. IC 10 has a very high SFR surface density, but only a relatively small advection speed. This outlier may point to the limitations of a scaling with $\Sigma_{\text {SFR }}$. The advection speed scales also with the rotation speed of the galaxy as $v \propto v_{\text {rot }}^{1.4}$ (Fig. 18(c)). The fact that the advection speed is related to the SFR surface density may be a consequence of a supernovae-driven blast wave (Vijayan et al. 2020). In contrast, for cosmic ray-driven wind models, or for any other wind model, the advection speed is expected to scale with the escape velocity as long as gravity is included (Ipavich 1975; Breitschwerdt et al. 1991; Everett et al. 2008), so the scaling with rotation speed is expected as well. Including IC 10 gives an indication that a wind model is preferred, but clearly more dwarf irregular galaxies need to be studied. 


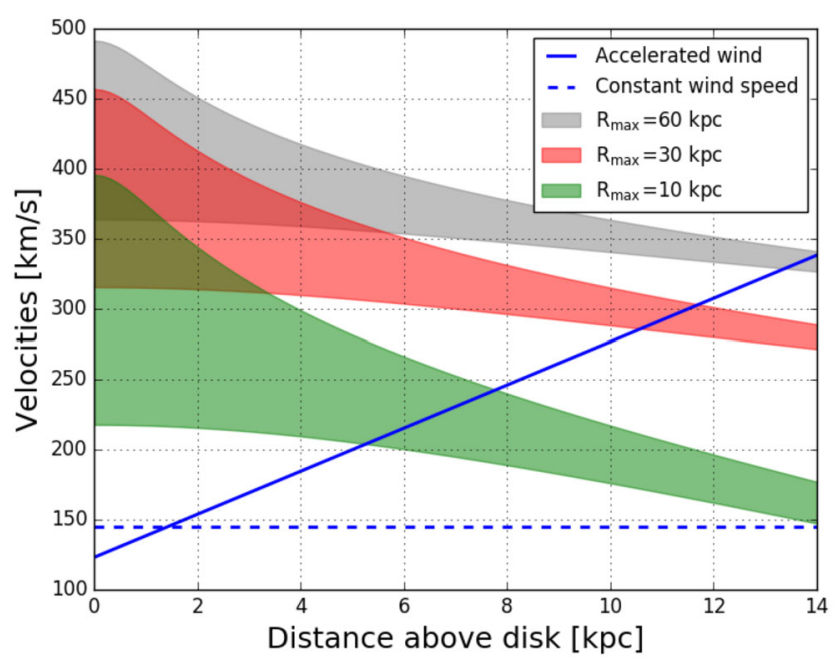

Fig. 19 Advection speed in NGC 3556 (M 108) as an example for advection-dominated $\mathrm{CR} e^{-}$transport with an accelerating wind. Shaded ares show the expected escape velocity for different dark matter halo distributions. The dashed line shows the best-fitting model with a constant advection speed for comparison. From Miskolczi et al. (2018)

\subsection{Accelerated advection speed}

With the advent of LOFAR, we are now able to probe the areas in the halo far away from the star-forming midplane with height the excess of $10 \mathrm{kpc}$, where we can probe compatibility of our data with accelerating winds. Miskolczi et al. (2018) have shown that in the galaxy NGC 3556 (M 108) an accelerating wind fits better than advection with a constant wind speed, where they assumed a linearly accelerating wind accelerating from $123 \mathrm{~km} \mathrm{~s}^{-1}$ near the midplane to $350 \mathrm{~km} \mathrm{~s}^{-1}$ at $14 \mathrm{kpc}$ distance (see Fig. 19). This is the first time, where an accelerating wind fits better, whereas with GHz-observations a constant wind speed fits equally well as an accelerating wind (Schmidt et al. 2019). An accelerating wind has the advantage that one can have energy equipartition between the cosmic rays and the magnetic field in the halo. For instance, Mora-Partiarroyo et al. (2019a) have shown that a constant advection speed can lead to a divergence between the cosmic-ray energy and the magnetic field of up to a factor of 40 in the halo. For an accelerating wind, cosmic rays can be in equipartition with the magnetic field and possibly even with the warm neutral and the warm ionised gas (see Fig. 20), which is physically more plausible.

Several possible advection profiles were investigated by Miskolczi et al. (2018), where they parametrised the advection velocity using equation (26). For $\beta=1$, the wind is a linearly accelerating, for $\beta=0.5$ the wind acceleration is high near the disc and then tailors off in the halo. They found that $\beta=1$ fits best to the observations. Schmidt et al. (2019) also use a linear advection velocity profile successfully. Hence, a linear advection speed profile appears to be

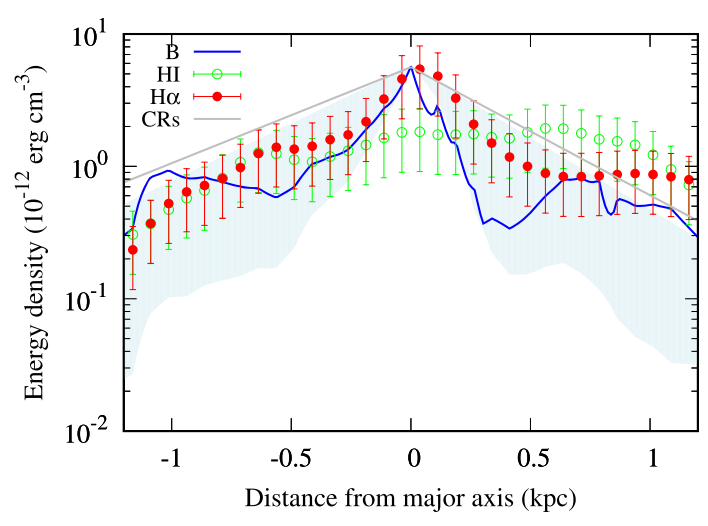

Fig. 20 Vertical profiles of the energy densities in the dwarf irregular galaxy IC 10 of the magnetic field $(B)$, the warm neutral medium $(\mathrm{H} \mathrm{I})$, the warm ionised medium $(\mathrm{H} \alpha)$, and the cosmic rays (CRs). From Heesen et al. (2018a)

favoured by observations thus far. In Schmidt et al. (2019), the local advection speed was investigated as well. Surprisingly, the advection is smaller in the centre of the galaxy. This is in contrast to the stronger gravitational acceleration in the centre of the galaxy should lead to higher advection speeds as Breitschwerdt et al. (2002) demonstrated for the case of the Milky Way.

\section{Stellar feedback-driven wind}

Thus far we have used the $\mathrm{CR} e^{-}$as tracers for a galactic wind and neglected the dynamical influence that the cosmic rays have themselves on the wind. Together with the thermal gas they may be able to drive a wind as a result of stellar feedback as is now widely accepted in the literature (e.g. Ipavich 1975; Breitschwerdt et al. 1991; Everett et al. 2008; Recchia et al. 2016; Mao and Ostriker 2018). In this section, we present a simple approach that tries to emulate such a wind model, but sidestepping the details of cosmicray transport which is needed to create such a wind in the first place. For the latter, it is usually assumed that either diffusion or streaming in addition to advection is needed to prevent the adiabatic cooling of the wind. Without such detailed modelling it is not possible to distinguish between the dynamical influence of the thermal and cosmic-ray gas, hence we refer this model to as generic 'stellar feedback-driven wind'. Nevertheless, our approach already fulfils some of the requirements we identified in Sect. 7:

- (i) advection speed is a 'wind solution';

- (ii) energy equipartition between cosmic rays and the magnetic field;

- (iii) linearly increasing advection speed.

Assumption (i) is motivated by the fact that a tight correlation between advection speed an escape velocity (i.e. rotation velocity) is observed (Sect. 7.4). Assumption (ii) is 
made such that energy equipartition is required as suggested by the tight radio-SFR relation (Sect. 6.2). The magnetic fields should be approximately exponential since that is the shape of the vertical intensity profiles (Sect. 4.1.1). Assumption (iii) fulfils our finding that linear profiles are well fitting the LOFAR data (Sect. 7.5). We attempt to meet these requirements with a simple iso-thermal wind model.

\subsection{Motivation}

We assume that the cosmic rays are advected in the flow of magnetised plasma, which is directed vertically and expands adiabatically. We use the following functional term for the cross-sectional area:

$A(z)=A_{0}\left[1+\left(\frac{z}{z_{0}}\right)^{\beta}\right]$,

which describes the 'flux tube' geometry. It has been widely used in semi-analytic 1D cosmic ray-driven wind models (Breitschwerdt et al. 1993; Everett et al. 2008; Recchia et al. 2016). This choice eases the comparison with these aforementioned models. If $\beta=2$ then the model is an expanding cone with a constant opening angle. We may possibly identify these flux tubes with the bubble-like features that definitely play a role as well and disc-halo interface may be more akin to a 'boiling disc' found in radio continuum observations (Stein et al. 2020) but also in simulations (Krause et al. 2021). Once these bubbles break out of the thin gaseous disc, the field lines open up and a chimney is formed (Norman and Ikeuchi 1989). These bubbles and chimneys then may merge and form together a kpc-sized superbubble that expands further into the halo, something that is suggested by the properties of warm dust in the halo (Yoon et al. 2021). The boundary of such a bubble may be related to the Xshaped structures centred on the nucleus, but with footpoints at a galactocentric radius $r_{0}$. Thus then would define the midplane flow radius, which may be the boundary of this outflow (Veilleux et al. 2021, see also Fig. 21). We now also need an equation that governs the magnetic field strength:

$B=B_{0}\left(\frac{r_{0}}{r}\right) \times\left(\frac{v_{0}}{v}\right)$,

where $B_{0}$ is the magnetic field strength in the galactic midplane, and $r_{0}$ and $v_{0}$ are the mid-plane flow radius and advection speed, respectively. This is the expected behaviour for radial and toroidal magnetic field components in a quasi1D flow (Baum et al. 1997). Since we do not take rotation into account, we cannot include any dynamical effect that the magnetic field might have on the wind (see Steinwandel et al. 2020, for a simulation of a magnetically driven wind). The continuity equation needs to be fulfilled:

$\rho v A=$ const.

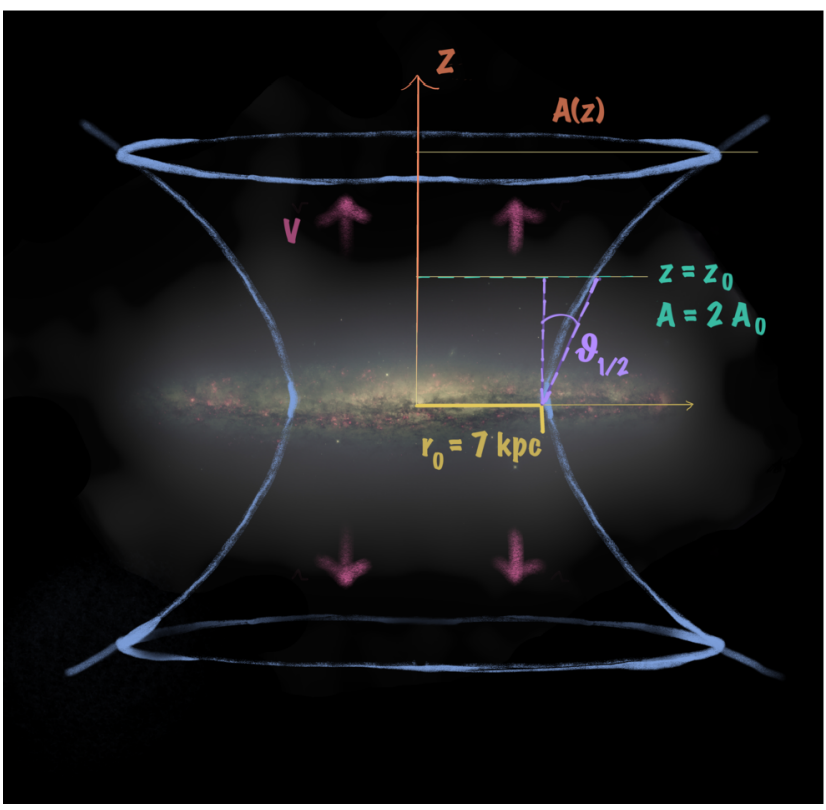

Fig. 21 Outflow geometry for the stellar feedback-driven wind model. The conical expanding cross-section can be described by the flux tube approximation. From Heald et al. (2021)

where $v$ is the advection speed and $\rho$ is the gas density. The momentum conservation is governed by the Euler equation:

$\rho v \frac{\mathrm{d} v}{\mathrm{~d} z}=\frac{\mathrm{d} P}{\mathrm{~d} z}-g \rho$,

where $P$ is the combined cosmic-ray and gas pressure and $g$ is the gravitational acceleration. With such a setup, we obtain approximate energy equipartition. Integrating the Euler equation leads to a wind equation, where we assume for simplicity that the compound sound speed $v_{\mathrm{c}}^{2}=P / \rho$ is constant. It can be shown that the wind velocity profile is in linear approximation:

$v=v_{\mathrm{c}}\left(1+\frac{z-z_{\mathrm{c}}}{z_{0}}\right)$

where $z=z_{\mathrm{c}}$ is the so-called critical point of the wind solution and $v=v_{\mathrm{c}}$ is the velocity at the critical point equivalent to the compound sound speed (Heald et al. 2021). This means we can parametrise the wind velocity profile in a linear way as required.

As we do not solve the energy equation explicitly, we have to check whether the energy conservation is indeed fulfilled. This is done via a cloud entrainment factor $\epsilon$, where the total energy flux in the wind is limited by the cosmicray luminosity (equation (1)) $L_{\mathrm{CR}}=1 / 2 \epsilon \dot{M} v^{2}$ with $\dot{M}$ the global mass-loss rate. This entrainment factor is expected to be of order unity for a cosmic ray-driven wind. 


\subsection{Application to NGC 5775}

The model is applied to LOFAR 150-MHz and CHANGES 1.5-GHz observations of NGC 5775 (Heald et al. 2021). The data can be indeed well fitted, with a linear acceleration of the advection speed as a result of the wind model (equation (32)) and an expanding bi-conical outflow (see Fig. 21). Using the compound sound speed, the thermal electron densities can be calculated. The electron density decreases from a few $10^{-3} \mathrm{~cm}^{-2} \mathrm{~s}^{-1}$ by a factor of 10 at the detection limit of the halo at $z \approx 15 \mathrm{kpc}$. This phase seems to be most consistent with the hot ionized medium (HIM). There are indications that in certain places the warm ionized medium (WIM) may be entrained in certain places, in particular near the $\mathrm{H} \alpha$ filaments (Tüllmann et al. 2000). The implied massloss rate $\dot{M}$ is a few solar masses per year. As the advection speeds exceeds the escape velocity at the edge of the halo, it is suggested that the mass is lost entirely from the galaxy. The mass-loss efficiency $\eta=\dot{M} / S F R$ would then be of order unity. However, we point out that there is substantial uncertainty arising from the outflow geometry and the poorlyunderstood distribution of ISM material entrained in the vertical flow.

\section{Spectroscopic observations}

\subsection{Wind speed}

The arguably most direct way to identify outflows and measure outflow speeds are spectroscopic observations. In the optical wavelength range, the interstellar $\mathrm{Na}$ I absorption line can be used (Martin 2005; Rupke et al. 2005), although the drawback of this particular line is that it works only in galaxies at the higher end of the luminosity scale. This limitation was remedied with the Cosmic Origins Spectrograph (COS) aboard the Hubble Space Telescope (HST), which made it possible to use ultraviolet absorption lines such as of Si II (Chisholm et al. 2015) and C II, Si III, Si IV, and N II (Heckman et al. 2015; Heckman and Borthakur 2016). These data trace the warm ionized phase, which is supposed to carry the bulk of the mass in an outflow and so allows us to trace winds in normal star-forming galaxies. This phase can be also seen in emission using the $\mathrm{H} \alpha$ line, but this again requires high SFRs, so that the galaxies are classified as (U)LIRGs (Arribas et al. 2014).

Our advection speeds increase with the SFR, $\Sigma_{\text {SFR }}$ and rotation speed $v_{\text {rot }}$, which indicates that they are tracing stellar feedback-driven winds (Sect. 7.4). We now compare the advection speed scaling relations (Table 3 ) with the equivalent relation of the gaseous tracers. The UV-absorption line measurements by Chisholm et al. (2015) point to a weak dependence of the outflow speed with the SFR of $v \propto S F R^{0.08-0.22}$ and similarly with the rotation speed of $v \propto v_{\text {rot }}^{0.44-0.87}$. In contrast, Heckman and Borthakur (2016), also using UV-absorption lines, find much stronger dependencies with $v \propto S F R^{0.32 \pm 0.02}$ and $v \propto v_{\text {rot }}^{1.16 \pm 0.37}$ (see also Heckman et al. 2015). Martin (2005) used Na I and K I absorption lines in ultra-luminous infrared galaxies and found $v \propto S F R^{0.35}$.

On the subject of whether the wind speed depends on $\Sigma_{\mathrm{SFR}}$, the literature is even more divided. Chisholm et al. (2015) did find no notable correlation, whereas Davies et al. (2019) claim a strong correlation of $v \propto \Sigma_{\mathrm{SFR}}^{0.34 \pm 0.10}$. Notably, the sample of Davies et al. (2019) contains mostly star bursts with $\Sigma_{\mathrm{SFR}}=0.1-1 \mathrm{M}_{\odot} \mathrm{yr}^{-1}$, whereas the sample by Chisholm et al. (2015) covers also lower values of $\Sigma_{\text {SFR }}$. Heckman and Borthakur (2016) claimed a correlation of $v \propto \Sigma_{\mathrm{SFR}}^{0.34}$ up to a value of $100 \mathrm{M}_{\odot} \mathrm{yr}^{-1}$, flattening out at even higher values. In Fig. 18, we compare the UV measurements of Heckman et al. (2015) with our advection speeds. In general, we find a good agreement with their wind speeds as function both of the SFR and rotation speed, although the scatter is fairly large for the UV measurements. For the comparison with the SFR surface density, there is no such good agreement, with our winds happening at much lower values of $\Sigma_{\mathrm{SFR}}$. In part this may be explained by our different definition of $\Sigma_{\text {SFR }}$, which employs the full extent of the star-forming disc whereas Heckman et al. (2015) use an effective (half-light) star-forming disc radius.

\subsection{Mass loading}

The mass-loading factor is defined as $\eta=\dot{M} / S F R$, where $\dot{M}$ is the mass-loss rate. The mass-loading factor is predicted to increase strongly with decreasing rotation speed, so that in dwarf galaxies the mass-loading factor could easily exceed unity, whereas in Milky Way-type $L_{\star}$ galaxies, the factor is of order unity. Chisholm et al. (2017) parametrised the mass-loading factor as:

$\eta=1.12 \pm 0.27\left(\frac{v_{\text {rot }}}{100 \mathrm{~km} \mathrm{~s}^{-1}}\right)^{-1.56 \pm 0.25}$,

using UV-absorption line studied of outflows. Similarly, Heckman and Borthakur (2016), also using UV-absorption line studies, found a slightly flatter dependency of $\eta \propto$ $v_{\text {rot }}^{-0.98}$. While we have not applied our stellar feedbackdriven wind model (Sect. 8) to a sample yet, we can use the theoretical expectation of a similar cosmic ray-driven wind model of $\eta \propto v_{\text {rot }}^{-5 / 3}$ (Mao and Ostriker 2018), which gives quite reasonable agreement. It is also encouraging that our one data point for NGC 5775 (Sect. 8.2) predicts a massloading factor of order unity, which is in good agreement with equation (33). 


\subsection{Wind velocity profile}

Wind velocity profile measurements are only few and far between since it requires spatially resolved line observations. The wind velocity profiles from the optical measurements look significantly differently than linear acceleration, where the acceleration happens close to the disc and converges quickly Chisholm et al. (2016). Notably, the acceleration happens already largely within $1 \mathrm{kpc}$ from the star burst region. Chisholm et al. (2016) attribute this velocity profile to either radiation pressure or cosmic-ray pressure, assuming that the accelerating force falls of with distance squared. There are a handful of other galaxies where the wind velocity profile has been measured such as in NGC 253 (Westmoquette et al. 2011), where outflow speeds of a few hundred $\mathrm{km} \mathrm{s}^{-1}$ are found within a few $100 \mathrm{pc}$ from the disc and which increase linearly with height.

Our radio haloes may require acceleration in particular if the lateral expansion needs to be limited as the morphology of the radio haloes suggests. On the other hand, the wind models such as of Chevalier and Clegg (1985) even with the inclusion of cosmic rays (Samui et al. 2010; Yu et al. 2020) all predict rapid acceleration near the disc even when adopted to the flux tube geometry (Heald et al. 2021). Hence, the jury is still out whether the wind velocity profiles are more in agreement with a linear acceleration across the size of the halo $(\sim 10 \mathrm{kpc})$, possibly extending even further, as some wind models predict that do not include an extended area of mass-loading but inject all energy at $z=0 \mathrm{kpc}$ Breitschwerdt et al. (1991), Everett et al. (2008), Recchia et al. (2016). While using the radio spectral index is a rather indirect way of measuring the velocity profile and subject to assumptions about the magnetic field, some form of acceleration seems to be most plausible as it is also the result of any stellar feedback-driven wind model (Sect. 8).

\subsection{Outflow size}

The outflow size in most absorption line studies is only a few $\mathrm{kpc}$ at most (Heckman and Borthakur 2016), whereas radio haloes are indicative of galaxy-wide outflows with radii typically a few kpc. Although the boundary of radio haloes is poorly defined, but the size of the haloes is typically comparable to the size of the star-forming disc (Dahlem et al. 2006). The connection of the galaxy-wide outflows with nuclear star bursts is rather uncertain (Westmoquette et al. 2011). A case in point is NGC 253, which does have a nuclear star burst with $\Sigma_{\mathrm{SFR}} \sim 1 \mathrm{M}_{\odot} \mathrm{yr}^{-1} \mathrm{kpc}^{-2}$, which shows a well-defined nuclear outflow (Heesen et al. 2011). The same galaxy has also a galaxy-wide advective radio halo (Heesen et al. 2009) and an X-ray halo indicating a galaxywide outflow as well (Bauer et al. 2008). It is possible that the 'down the barrel' optical and UV spectroscopic surveys do overlook the larger size of the outflow region due to sensitivity issues since a broad component emission or absorption line has to be identified.

When other measurement are used such integral field unit (IFU) spectroscopy, the size of the haloes are much larger in width. In the SAMI data of Ho et al. (2016), the velocity field is widely asymmetric in galaxies indicating a larger outflow size. In the CALIFA sample, López-Cobá et al. (2019) identified outflows with increasing line ratios such as $[\mathrm{N} \mathrm{II}] / \mathrm{H} \alpha$ along the semi-major axis. Such increasing ratios are consistent with shock ionization in galactic outflows. Again, the morphology points to galactic outflows.

\subsection{Outflow threshold}

The existence of a minimum value for the star-formation rate surface density was first posed by Rossa and Dettmar (2003b), who studied the extra-planar diffuse ionized gas (eDIG) in edge-on galaxies. Their value is $\Sigma_{\mathrm{SFR}}=2 \times$ $10^{-3} \mathrm{M}_{\odot} \mathrm{yr}^{-1} \mathrm{kpc}^{-2}$, which was then corroborated by Tüllmann et al. (2006) who studied extra-planar hot ionized gas via $\mathrm{X}$-ray emission. In most galaxies, there is no extended eDIG emission detected below this threshold, and if there is a detection, the dust temperature is significantly higher. This threshold is much lower than the canonical threshold for galactic winds by Heckman et al. (2000), who suggested $\Sigma_{\mathrm{SFR}} \approx 10^{-1} \mathrm{M}_{\odot} \mathrm{yr}^{-1} \mathrm{kpc}^{-2}$. Galaxies exceeding this value are commonly referred to as 'superwind' galaxies and are known to have extensive X-ray haloes (Strickland et al. 2004). More recent observations have shown this outflow threshold to be potentially much lower, as for instance the detection of a superbubble of warm dust in NGC 891 with a local $\Sigma_{\mathrm{SFR}}$ of $0.03 \mathrm{M}_{\odot} \mathrm{yr}^{-1} \mathrm{kpc}^{-2}$ suggests (Yoon et al. 2021). It is probably the local value of $\Sigma_{\text {SFR }}$ which needs to be $\sim 10^{-2} \mathrm{M}_{\odot} \mathrm{yr}^{-1} \mathrm{kpc}^{-2}$ to allow the formation of chimneys facilitating outflows. The chimneys would form at spiral arms and predominantly at smaller galactocentric radii allowing an outflow in the inner parts of the galaxy (see instructive simulations by Krause et al. 2021).

Ho et al. (2016) suggests much lower values of globally averaged star formation rates with $\Sigma_{\mathrm{SFR}}=S F R /\left(\pi r_{\mathrm{e}}^{2}\right)$ with values of $10^{-3}-10^{-1.5} \mathrm{M}_{\odot} \mathrm{yr}^{-1} \mathrm{kpc}^{-2}$, where $r_{\mathrm{e}}$ is the effective radius. López-Cobá et al. (2019) suggest $\Sigma_{\text {SFR }}>$ $10^{-2} \mathrm{M}_{\odot} \mathrm{yr}^{-1} \mathrm{kpc}^{-2}$ in conjunction with a centrally concentrated gas distribution. Clearly, this value depends on the detection method. The radio continuum method suggests also a low threshold of around the value as for the existence of eDIG. This threshold is identified by the transition from diffusion-dominated haloes to advection-dominated ones (Sect. 4.1 and Fig. 18(b)). That this property of radio haloes fits to the optical observations raises the possibility that the vertical profile of radio haloes (Gaussian and exponential, Sect. 4.1) and the multi-component fitting (Sect. 4.1.3) allow us already to distinguish between galaxies with outflows and those that do not have outflows. 


\subsection{Cosmic-ray calorimetry}

The detection of $\gamma$-rays from star-forming galaxies with Fermi has allowed us to compare the $\gamma$-ray luminosity with expectations from cosmic-ray calorimetry (Ackermann et al. 2012). Lacki et al. (2011) show that the star-burst nuclei of NGC 253 and M 82 are closest to calorimetry, although there is some uncertainty with regards to the $\mathrm{GeV}$-emission from secondary $\mathrm{CR} e^{-}$. Yoast-Hull et al. (2013) showed that M 82 is a good electron calorimeter but not proton calorimeter, while for NGC 253 the situation is more complex (YoastHull et al. 2014). It has been recently suggested by Hopkins et al. (2020) that the other galaxies at lower SFRs are only poor cosmic-ray proton calorimeters. This requires a fast escape of cosmic rays either by diffusion, as facilitated with a high diffusion coefficient (Hopkins et al. 2020), or via a galactic wind.

\subsection{Extra-planar gas}

The extra-planar gas comprises several phases, the warm neutral medium traced by $\mathrm{HI}$, the WIM traced by $\mathrm{H} \alpha$, and the HIM traced by X-ray emission. The HIM as traced by $\mathrm{X}$-ray emission has electron density scale heights between 4 and $8 \mathrm{kpc}$ with exponential profiles preferred over Gaussian or power-law profiles (Strickland et al. 2004; HodgesKluck and Bregman 2013). These data would be in approximate agreement with what is expected for an outflow of a hot wind. In contrast, the WIM as traced by $\mathrm{H} \alpha$ emission has much smaller scale heights of $\sim 1 \mathrm{kpc}$ (Dettmar and Soida 2006), although in places there can be filaments with much larger scale heights of 3-5 kpc (Boettcher et al. 2013). These profiles can be again approximated by exponential functions. The atomic gas as traced by $\mathrm{H} \mathrm{I}$ emission has a large variety of scale heights (Zschaechner et al. 2015), sometimes with both a thin and thick in the order of between a few $100 \mathrm{pc}$ and a few $\mathrm{kpc}$.

Because both the $\mathrm{HI}$ and $\mathrm{H} \alpha$ are line emissions, one can measure the rotation speed of edge-on galaxies as function of height. It is observed that the rotation speed of the gas decreases approximately linearly with height. This is referred to as 'rotational lag' and has an amplitude of 5-20 $\mathrm{km} \mathrm{s}^{-1} \mathrm{kpc}^{-1}$ (Heald et al. 2006; Zschaechner et al. 2015).

\section{Inferences from theory}

\subsection{Cosmic ray-driven wind models}

For cosmic rays to able to drive a wind, they have to be effectively confined in the galaxy for some time. We recall that the cosmic-ray mean free path is only a few pc, so that galaxies are effectively optically thick for cosmic rays. The cosmic rays then transfer a small part of their momentum and energy on the gas every time they interact via Alfvén waves, which they generate themselves via the streaming instability ('self-confinement' picture Zweibel 2013). The 1D cosmic ray-driven wind models by Breitschwerdt et al. (1991) and Everett et al. (2008) showed that cosmic rays streaming along the magnetic field lines can compensate for the adiabatic cooling of the wind fluid and so the compound sound speed increases slightly in the halo. This is required for a wind solution to go through the critical point where the gravitational acceleration is approximately constant as in the case for a galaxy halo (Mao and Ostriker 2018). The wind velocity profiles are approximately linear before the speed converges to a few times the rotation speed (Everett et al. 2008). One of the limitations of these wind models is that all the energy and mass are injected at $z=0$, which is not very physical. The widely used analytical wind model of Chevalier and Clegg (1985) has a driving region where the energy and momentum are injected, which is more realistic. This model was extended by Samui et al. (2010) to include cosmic rays, which shows rapid acceleration in the driving region and nearly constant velocity at larger radii. As the driving region is rather confined to the disc plane with a height of $\sim 100 \mathrm{pc}$, similar to the gaseous scale height of the warm neutral medium, the acceleration will be small at heights $\gtrsim 1 \mathrm{kpc}$.

In a steady state and without considering internal losses, the cosmic rays would transfer a fraction of their luminosity on the total energy flux in the wind, so $L_{\mathrm{CR}} \approx$ $1 / 2(\epsilon / 0.5) \dot{M} v^{2}$, where $\epsilon$ is an efficiency factor. As we shown in Sect. 8, this is consistent with a stellar feedbackdriven wind model in NGC 5775. A consequence of cosmic ray-driven winds is that the hydrodynamical equilibrium state of galaxies will be affected by the cosmic rays (Crocker et al. 2020). Another property of cosmic ray-driven wind models, which include gravity, is that the wind velocity scales linearly with the rotation speed in the galaxy (Ipavich 1975). Such a behaviour is also expected for 'momentumdriven' winds for which radiative cooling is important (Murray et al. 2005; Veilleux et al. 2020). The advection speed scaling relation (Sect. 7.4) with the rotation speed is in good agreement with such an expectation.

\subsection{Simulations}

Simulations have shown how important cosmic rays are in order to create galaxy-wide outflows. There are now many MHD simulations available that include cosmic rays such as the one of Girichidis et al. (2018). They showed that cosmic ray-driven outflows are significantly cooler with a large fraction of the gas staying at $10^{4} \mathrm{~K}$ rather than $10^{6} \mathrm{~K}$ for the thermally driven case. The reason that cosmic rays are so effective at driving galactic winds is that they can diffuse out 
Table 4 Cosmic ray (CR)-driven 1D wind models

\begin{tabular}{lllllll}
\hline Model & Driving & Diffusion & Streaming & Gravity & Rotation & Reference \\
\hline aConstant wind speed & N/A & $\times$ & $\times$ & $\times$ & $\times$ & Heesen et al. (2018b) \\
a Accelerated wind & N/A & $\times$ & $\times$ & $\times$ & $\times$ & Miskolczi et al. (2018) \\
b Iso-thermal wind & hybrid & $\times$ & $\times$ & $\checkmark$ & $\times$ & Heald et al. (2021) \\
CR-driven wind with streaming & hybrid & $\times$ & $\checkmark$ & $\checkmark$ & $\times$ & Everett et al. (2008) \\
CR-driven wind with diffusion & hybrid & $\checkmark$ & $\checkmark$ & $\checkmark$ & $\times$ & Recchia et al. (2016) \\
CR-driven wind with rotation & hybrid & $\times$ & $\checkmark$ & $\checkmark$ & $\checkmark$ & Zirakashvili et al. (1996) \\
CR-driven wind w. rot. \& diff. & hybrid & $\checkmark$ & $\checkmark$ & $\checkmark$ & Ptuskin et al. (1997) \\
\hline
\end{tabular}

Note: Hybrid driving means both a dynamical influence of the thermal gas (HIM) and the cosmic rays

${ }^{\mathrm{a}} \mathrm{CR} e^{-}$are only used as tracers with no dynamical influence of the cosmic rays

${ }^{\mathrm{b}} \mathrm{CR} e^{-}$are used as tracers with a simplified iso-thermal wind model that includes cosmic-ray pressure (Sect. 8)

of star-forming regions and then create a 'background sea' with a pressure gradient on kpc-scales (Salem and Bryan 2014). This gradient then can lift the gas into the halo. The transport of cosmic rays is important as if the cosmic rays are just advected with the gas, they act only as an additional pressure component and so the gaseous disc is 'puffed up', but no outflow is created (Ruszkowski et al. 2017).

The cosmological simulations by Pakmor et al. (2016b) showed the influence of anisotropic cosmic-ray diffusion. Galaxies with spiral magnetic fields created in part by differential rotation, can store cosmic rays for longer. While a wind develops both in the case of anisotropic and isotropic diffusion, the isotropic diffusion suppresses the magnetic field amplification in the disc. Again, without diffusion, a wind does not form at all. In Jacob et al. (2018), simulations of dwarf galaxies show spherical winds with slow speeds of $20 \mathrm{~km} \mathrm{~s}^{-1}$, whereas galaxies with higher masses have more bi-conical outflows with higher speeds of $200 \mathrm{~km} \mathrm{~s}^{-1}$. Interesting, for galaxies in excess of a virial mass of $10^{11.5} \mathrm{M}_{\odot}$ do not form cosmic ray-driven winds beyond the virial radius. For all cases, the outflow speed is in good agreement with the escape velocity near the galactic mid-plane. However, their wind speeds seem to be always on the low side when compared with optical observations and our data.

\subsubsection{Wind velocity profiles}

The simulations by Girichidis et al. (2018) showed approximately linearly accelerating mass-weighted velocity profiles. They simulated only the first $2 \mathrm{kpc}$ near the galactic mid-plane, so that it is not clear whether the escape velocity is reached. The authors point out that further acceleration is expected in the halo. The outflow speeds they find are $\sim 50 \mathrm{~km} \mathrm{~s}^{-1}$, so significantly lower than what we measure near the disc. In MHD simulations of isolated galaxies with cosmic rays, Jacob et al. (2018) found that the vertical velocity profiles are in good agreement with the wind model of Chevalier and Clegg (1985), with rapid acceleration near the disc and then a nearly constant velocity in the halo.

\subsubsection{Cloud entrainment}

The entrainment of clouds is important to load the wind, which contains mostly of the HIM, with further mass. Banda-Barragán et al. (2020) showed that the hot wind is able to accelerate clouds at a speed comparable to the escape velocity within the first $1 \mathrm{kpc}$ away from the disc. These clouds form a 'mist' of WIM that can be further accelerated in the wind. The wind is formed this close to the disc as expanding superbubbles which are particularly good in converting the kinetic energy of $\mathrm{SNe}$ into thermal energy, i.e. the thermalization efficiency is quite high with a few 10 per cent (Sharma et al. 2014). What appears to be important is that these clouds can then be considered as the starting point for the reference level at $1 \mathrm{kpc}$ where we start to see advection-dominated haloes. It appears hence as possible that these clouds are advected as a thin mist with the fast flow of the HIM and traced by the $\mathrm{CR} e^{-}$.

As noted in Sect. 9.1, there is a good agreement between the scaling relations as found for the advection speeds and the cool neutral and warm ionized outflows as measured from UV interstellar absorption lines. This is the case for both the magnitude of the outflow velocity as well as the scaling relations slopes. This agreement, while assuming that radio traces rather the HIM (Sect. 8.2), needs entrainment of clouds in order to get similar wind speeds for the clouds.

\section{Missing physics}

We have now approached a phase where we have demonstrated that radio continuum observations can give us a complementary view on galactic winds. What we have not yet 
been able to do though is to identify the mechanisms that allow us to probe the workings of a cosmic ray-driven galactic wind. In our iso-thermal wind model we have only assumed that the cosmic rays do not cool adiabatically and the sound speed is constant (Sect. 8). This can be achieved both by either cosmic-ray streaming along magnetic field lines or anisotropic diffusion. This has been achieved already with 1D models in the literature, so their application remains to be carried out in future work. In Table 4, we present an overview of our pure phenomenological models and the 1D wind models from the literature. We now discuss the main physical effects.

\subsection{Cosmic-ray streaming}

The vertical spectral index profiles are well fitted with our advection models, although our concave model profiles may still be improved in order to fit the data better (Miskolczi et al. 2018). This would require a faster $\mathrm{CR} e^{-}$bulk speed without the change in magnetic field as demanded by the continuity equation and energy equipartition. Such a change may hence be in agreement with cosmic-ray streaming which does change the $\mathrm{CR} e^{-}$bulk speed. As it was also suggested, the streaming speed can be even a few times the Alfvén speed, when the cosmic rays decouple from the magnetic field such as when neutral atoms suppress Alfvén waves (Ruszkowski et al. 2017). This raises the possibility to detect a particularly fast cosmic-ray bulk speed in areas with an excess of $\mathrm{H}$ I emission.

An alternative suggestion is to measure the velocity differential between the $\mathrm{CR} e^{-}$bulk speed and the ionized gas in the outflow. For edge-on galaxies, the line-of-sight velocity is of course fairly small depending on the outflow opening angle. The entrained clouds should soon reach the speed of the HIM, so that we can use these clouds as a tracer for the outflow speed (Banda-Barragán et al. 2020). The global wind speeds appear to be in good agreement with what has been measured for the WIM using optical and UV spectroscopy (Sect. 9.1), but there may still be local effects in particular where the magnetic field has a strong vertical component (Tüllmann et al. 2000).

\subsection{Cosmic-ray diffusion}

Near the galactic mid-plane, cosmic-ray diffusion is important. The high-angular resolution images resolved on a 1kpc scale resolve the thin radio disc. Hence, the escape of the cosmic rays is governed by the superposition and advection. Since we do not resolve this region well with our observations, we have neglected its influence. However, as Breitschwerdt et al. (1993) and Recchia et al. (2016) have shown, this region is quite important for the launching of the wind and also for the cosmic-ray spectrum (Ptuskin et al.
1997). Obviously, the escape of $\mathrm{CR} e^{-}$would have a bearing also on the integrated radio spectral index, which we can measure now over several decades in frequency. As pointed out before, while we may neglect diffusion in comparison to advection for simply measuring the transport of $\mathrm{CR} e^{-}$ (Sect. 2.2.1), from a theoretical point of view we cannot trace the makings of a cosmic ray-driven wind without taking diffusion into account.

\subsection{Rotation}

We have neglected the influence of the magnetic pressure on the outflow velocity. This is the case if the outflow follows the magnetic field lines, so that lines of magnetic force are parallel to the wind velocity. Obviously, this will have some consequence for the outflow geometry which we can potentially test with linear polarisation measurements. Due to the superposition of the azimuthal velocity and vertical velocity, the magnetic field lines would wind up in a helical shape in the halo. This may be potentially observable as a rotation measure signal. So far linear polarisation measurements have shown X-shaped magnetic fields but only little or no large-scale rotation measure signal in the halo (Soida et al. 2011).

What then also needs to be taken into account is a proper treatment of the angular momentum in the outflow. This will change the wind solutions as Zirakashvili et al. (1996) have demonstrated. They also found that the dynamics of the ionized gas in the halo is changed since some of the angular momentum is transferred to this gas.

\section{Summary}

As we have demonstrated in this review, radio continuum observations open up a new window on cosmic-ray transport in nearby galaxies. These observations allow us to calibrate the influence that cosmic rays have on galactic winds, a process that shapes and influences galaxy evolution in a unique way. Cosmic rays are transported by diffusion, advection, and streaming, which all contribute to a different degree. Since galaxies have complex magnetic field configurations, they are effectively optically thick to the scattering of cosmic rays. With radio continuum observations we trace cosmic-ray electrons in $\mathrm{GeV}$-energy range, corresponding to the peak of the cosmic ray energy density in the spectrum of protons and heavier nuclei. In summary, our main results are as follows:

1. Diffusion coefficients for $\mathrm{GeV}$-cosmic rays are of order $10^{28} \mathrm{~cm}^{2} \mathrm{~s}^{-1}$, with either little or no energy dependence (Sect. 7.1); 
2. Gaussian radio haloes are diffusion-dominated and are found in galaxies with no winds, whereas exponential radio haloes are advection-dominated indicative of winds (Sect. 4.1);

3. Advective radio haloes are predominant in galaxies with $\Sigma_{\mathrm{SFR}} \geq 2 \times 10^{-3} \mathrm{M}_{\odot} \mathrm{yr}^{-1} \mathrm{kpc}^{-2}$ (Sect. 4.1.1); this suggests that there is a $\Sigma_{\mathrm{SFR}}$-threshold value for galactic winds (Sect. 9.5);

4. The advection speed scales with SFR, $\Sigma_{\mathrm{SFR}}$, and $v_{\text {rot }}$ (Sect. 7.4), corroborating stellar feedback as the cause for radio haloes;

5. The high advection speeds, comparable to the escape velocities, suggest that the gas can escape into the CGM and contribute to the escape of baryons and metals;

6 . The advection speed scaling relations are in good agreement with what has been measured using optical and UV spectroscopy, further corroborating galactic winds and radio haloes have a common cause (Sect. 9.1);

7. A stellar feedback-driven wind model suggests that the hot ionized medium is the main wind fluid (Sect. 8). If cosmic rays are driving the wind, the mass-loading factor could be of order unity for Milky Way-type galaxies. However, due to the uncertain geometry and entrainment of warm ionized and cold neutral medium clouds, this mass-loss rate might be substantially different.

There are a few caveats relevant to these conclusions, however. One of them is that it can be difficult to distinguish advection and diffusion based purely on the radio spectral index (Stein et al. 2019b). It is thus possible that galaxies near the diffusion-advection boundary may be misclassified. Another uncertainty is the unknown contribution from cosmic-ray streaming in edge-on galaxies, which may lead to advection speeds overestimating wind velocities. Hence, in the future a better modelling of streaming would be required, incorporating it into the stellar feedback-driven wind model (Sect. 7.2). In face-on galaxies, a better modelling of cosmic-ray diffusion and streaming while accounting for the escape of $\mathrm{CR} e^{-}$would be necessary in order to affirm measurements of the diffusion coefficient and to be able to distinguish between these transport modes (Sect. 6).

Our long-term goal is the aim to inform cosmological simulations of galaxies, which have to build in these kind of physics as part of 'subgrid' models (Vogelsberger et al. 2020 ). With new radio facilities now producing many observational data sets we can test these models, we expect that important input will come from observations for the foreseeable future.

Acknowledgements We would like to thank the editors of this Topical Collection, Manami Sasaki, Ralf-Jürgen Dettmar and Julia Tjus, for the invitation to write this review article. We also would like to thank the anonymous referee for an insightful report that helped to improve this paper. We thank our colleagues for providing important contributions to this article. In particular, we would like to thank Arpad Miskolczi, Yelena Stein, Philip Schmidt, Sarrvesh Sridahr, and George Heald.
They all helped a lot with studying the radio continuum observations and applying the SPINNAKER models to them. Arpad Miskolczi is also thanked especially for developing SPINTERACTIVE, which made the application of these models so much easier.

Funding Note Open Access funding enabled and organized by Projekt DEAL.

Data Availability All data generated or analysed during this study are included in this published article.

\section{Declarations}

Conflict of Interest The authors have no competing interests to declare that are relevant to the content of this article.

Open Access This article is licensed under a Creative Commons Attribution 4.0 International License, which permits use, sharing, adaptation, distribution and reproduction in any medium or format, as long as you give appropriate credit to the original author(s) and the source, provide a link to the Creative Commons licence, and indicate if changes were made. The images or other third party material in this article are included in the article's Creative Commons licence, unless indicated otherwise in a credit line to the material. If material is not included in the article's Creative Commons licence and your intended use is not permitted by statutory regulation or exceeds the permitted use, you will need to obtain permission directly from the copyright holder. To view a copy of this licence, visit http://creativecommons.org/licenses/by/4.0/.

\section{References}

Ackermann, M., Ajello, M., Allafort, A., Baldini, L., Ballet, J., Bastieri, D., Bechtol, K., Bellazzini, R., Berenji, B., Bloom, E.D., Bonamente, E., Borgland, A.W., Bouvier, A., Bregeon, J., Brigida, M., Bruel, P., Buehler, R., Buson, S., Caliandro, G.A., Cameron, R.A., Caraveo, P.A., Casandjian, J.M., Cecchi, C., Charles, E., Chekhtman, A., Cheung, C.C., Chiang, J., Cillis, A.N., Ciprini, S., Claus, R., Cohen-Tanugi, J., Conrad, J., Cutini, S., de Palma, F., Dermer, C.D., Digel, S.W., Silva, E.d.C.e., Drell, P.S., Drlica-Wagner, A., Favuzzi, C., Fegan, S.J., Fortin, P., Fukazawa, Y., Funk, S., Fusco, P., Gargano, F., Gasparrini, D., Germani, S., Giglietto, N., Giordano, F., Glanzman, T., Godfrey, G., Grenier, I.A., Guiriec, S., Gustafsson, M., Hadasch, D., Hayashida, M., Hays, E., Hughes, R.E., Jóhannesson, G., Johnson, A.S., Kamae, T., Katagiri, H., Kataoka, J., Knödlseder, J., Kuss, M., Lande, J., Longo, F., Loparco, F., Lott, B., Lovellette, M.N., Lubrano, P., Madejski, G.M., Martin, P., Mazziotta, M.N., McEnery, J.E., Michelson, P.F., Mizuno, T., Monte, C., Monzani, M.E., Morselli, A., Moskalenko, I.V., Murgia, S., Nishino, S., Norris, J.P., Nuss, E., Ohno, M., Ohsugi, T., Okumura, A., Omodei, N., Orlando, E., Ozaki, M., Parent, D., Persic, M., PesceRollins, M., Petrosian, V., Pierbattista, M., Piron, F., Pivato, G., Porter, T.A., Rainò, S., Rando, R., Razzano, M., Reimer, A., Reimer, O., Ritz, S., Roth, M., Sbarra, C., Sgrò, C., Siskind, E.J., Spandre, G., Spinelli, P., Stawarz, Ł., Strong, A.W., Takahashi, H., Tanaka, T., Thayer, J.B., Tibaldo, L., Tinivella, M., Torres, D.F., Tosti, G., Troja, E., Uchiyama, Y., Vandenbroucke, J., Vianello, G., Vitale, V., Waite, A.P., Wood, M., Yang, Z.: Astrophys. J. 755(2), 164 (2012). https://doi.org/10.1088/0004-637X/ 755/2/164. 1206.1346

Adebahr, B., Krause, M., Klein, U., Weżgowiec, M., Bomans, D.J., Dettmar, R.-J.: Astron. Astrophys. 555, 23 (2013). https://doi.org/ 10.1051/0004-6361/201220226. 1209.5552 
Ahlers, M., Mertsch, P.: Progress in Particle and Nuclear Physics 94, 184 (2017). https://doi.org/10.1016/j.ppnp.2017.01.004. 1612. 01873

Arribas, S., Colina, L., Bellocchi, E., Maiolino, R., Villar-Martín, M. Astron. Astrophys. 568, 14 (2014). https://doi.org/10.1051/00046361/201323324. 1404.1082

Banda-Barragán, W.E., Brüggen, M., Federrath, C., Wagner, A.Y., Scannapieco, E., Cottle, J.: Mon. Not. R. Astron. Soc. (2020). https://doi.org/10.1093/mnras/staa2904. 2009.07718

Basu, A., Beck, R., Schmidt, P., Roy, S.: Mon. Not. R. Astron. Soc. 449, 3879 (2015). https://doi.org/10.1093/mnras/stv510. 1503. 02420

Bauer, M., Pietsch, W., Trinchieri, G., Breitschwerdt, D., Ehle, M., Freyberg, M.J., Read, A.M.: Astron. Astrophys. 489, 1029 (2008). https://doi.org/10.1051/0004-6361:20078935. 0711.3182

Baum, S.A., O’Dea, C.P., Giovannini, G., Cotton, W.B., de Koff, S., Feretti, L., Golombek, D., Lara, L., Macchetto, F.D., Miley, G.K., Sparks, W.B., Venturi, T., Komissarov, S.S.: Astrophys. J. 483(1), 178 (1997). https://doi.org/10.1086/304221

Beck, R.: Astron. Astrophys. Rev. 24, 4 (2015). https://doi.org/10. 1007/s00159-015-0084-4. 1509.04522

Beck, R.: Astron. Astrophys. 578, 93 (2015). https://doi.org/10.1051/ 0004-6361/201425572. 1502.05439

Beck, R., Krause, M.: Astron. Nachr. 326, 414 (2005). https://doi.org/ 10.1002/asna.200510366. arXiv:astro-ph/0507367

Becker Tjus, J., Merten, L.: arXiv e-prints, 2002 (2020). 2002.00964

Bell, A.R.: Mon. Not. R. Astron. Soc. 182, 147 (1978). https://doi.org/ 10.1093/mnras/182.2.147

Berkhuijsen, E.M., Beck, R., Tabatabaei, F.S.: Mon. Not. R. Astron. Soc. 435, 1598 (2013). https://doi.org/10.1093/mnras/stt1400. 1307.7991

Boettcher, E., Zweibel, E.G., Yoast-Hull, T.M., Gallagher, J.S. III: Astrophys. J. 779, 12 (2013). https://doi.org/10.1088/0004-637X/ 779/1/12. 1311.0006

Braun, R., Oosterloo, T.A., Morganti, R., Klein, U., Beck, R.: Astron. Astrophys. 461, 455 (2007). https://doi.org/10.1051/0004-6361: 20066092. astro-ph/0610114

Breitschwerdt, D., McKenzie, J.F., Völk, H.J.: Astron. Astrophys. 245, 79 (1991)

Breitschwerdt, D., McKenzie, J.F., Völk, H.J.: Astron. Astrophys. 269, 54 (1993)

Breitschwerdt, D., Dogiel, V.A., Völk, H.J.: Astron. Astrophys. 385, 216 (2002). https://doi.org/10.1051/0004-6361:20020152. arXiv: astro-ph/0201345

Brüggen, M., Scannapieco, E.: Astrophys. J. 905(1), 19 (2020). https:// doi.org/10.3847/1538-4357/abc00f. 2010.07308

Buffie, K., Heesen, V., Shalchi, A.: Astrophys. J. 764, 37 (2013). https://doi.org/10.1088/0004-637X/764/1/37. 1212.5721

Caprioli, D.: J. Cosmol. Astropart. Phys. 2011(5), 026 (2011). https:// doi.org/10.1088/1475-7516/2011/05/026. 1103.2624

Chevalier, R.A., Clegg, A.W.: Nature 317(6032), 44 (1985). https://doi. org/10.1038/317044a0

Chisholm, J., Tremonti, C.A., Leitherer, C., Chen, Y., Wofford, A., Lundgren, B.: Astrophys. J. 811, 149 (2015). https://doi.org/10. 1088/0004-637X/811/2/149. 1412.2139

Chisholm, J., Tremonti Christy, A., Leitherer, C., Chen, Y.: Mon. Not. R. Astron. Soc. 463(1), 541 (2016). https://doi.org/10.1093/ mnras/stw1951. 1605.05769

Chisholm, J., Tremonti, C.A., Leitherer, C., Chen, Y.: Mon. Not. R. Astron. Soc. 469(4), 4831 (2017). https://doi.org/10.1093/mnras/ stx1164. 1702.07351

Chyży, K.T., Jurusik, W., Piotrowska, J., Nikiel-Wroczyński, B., Heesen, V., Vacca, V., Nowak, N., Paladino, R., Surma, P., Sridhar, S.S., Heald, G., Beck, R., Conway, J., Sendlinger, K., Curyło, M., Mulcahy, D., Broderick, J.W., Hardcastle, M.J., Callingham, J.R., Gürkan, G., Iacobelli, M., Röttgering, H.J.A., Adebahr, B., Shulevski, A., Dettmar, R.-J., Breton, R.P., Clarke, A.O.,
Farnes, J.S., Orrú, E., Pand ey, V.N., Pandey-Pommier, M., Pizzo, R., Riseley, C.J., Rowlinson, A., Scaife, A.M.M., Stewart, A.J., van der Horst, A.J., van Weeren, R.J.: Astron. Astrophys. 619, 36 (2018). https://doi.org/10.1051/0004-6361/201833133. 1808. 10374

Condon, J.J.: Annu. Rev. Astron. Astrophys. 30, 575 (1992). https:// doi.org/10.1146/annurev.aa.30.090192.003043

Crocker, R.M., Krumholz, M.R., Thompson, T.A.: arXiv e-prints, 2006 (2020). 2006.15821

Dahlem, M., Lisenfeld, U., Golla, G.: Astrophys. J. 444, 119 (1995). https://doi.org/10.1086/175587

Dahlem, M., Lisenfeld, U., Rossa, J.: Astron. Astrophys. 457, 121 (2006). https://doi.org/10.1051/0004-6361:20054787. arXiv: astro-ph/0607102

Davies, R.L., Förster Schreiber, N.M., Übler, H., Genzel, R., Lutz, D., Renzini, A., Tacchella, S., Tacconi, L.J., Belli, S., Burkert, A., Carollo, C.M., Davies, R.I., Herrera-Camus, R., Lilly, S.J., Mancini, C., Naab, T., Nelson, E.J., Price, S.H., Shimizu, T.T., Sternberg, A., Wisnioski, E., Wuyts, S.: Astrophys. J. 873(2), 122 (2019). https://doi.org/10.3847/1538-4357/ab06f1. 1808.10700

Dettmar, R.-J., Soida, M.: AN 327, 495 (2006). https://doi.org/10. 1002/asna.200610569

Dumas, G., Schinnerer, E., Tabatabaei, F.S., Beck, R., Velusamy, T., Murphy, E.: Astron. J. 141(2), 41 (2011). https://doi.org/10.1088/ 0004-6256/141/2/41. 1012.0212

Duric, N., Irwin, J., Bloemen, H.: Astron. Astrophys. 331, 428 (1998)

Enßlin, T., Pfrommer, C., Miniati, F., Subramanian, K.: Astron. Astrophys. 527, 99 (2011). https://doi.org/10.1051/0004-6361/ 201015652. 1008.4717

Everett, J.E., Zweibel, E.G., Benjamin, R.A., McCammon, D., Rocks, L., Gallagher, J.S. III: Astrophys. J. 674, 258 (2008). https://doi. org/10.1086/524766. arXiv:0710.3712

Farber, R., Ruszkowski, M., Yang, H.-Y.K., Zweibel, E.G.: Astrophys. J. 856(2), 112 (2018). https://doi.org/10.3847/1538-4357/ aab26d. 1707.04579

Girichidis, P., Naab, T., Hanasz, M., Walch, S.: Mon. Not. R. Astron. Soc. 479, 3042 (2018). https://doi.org/10.1093/mnras/sty1653. 1805.09333

Heald, G.H., Rand, R.J., Benjamin, R.A., Bershady, M.A.: Astrophys. J. 647(2), 1018 (2006). https://doi.org/10.1086/505464. astro-ph/0604588

Heald, G.H., Heesen, V., Sridhar, S.S., Beck, R., Bomans, D.J., Brüggen, M., Chyży, K.T., Damas-Segovia, A., Dettmar, R.-J., English, J., Henriksen, R., Ideguchi, S., Irwin, J., Krause, M., Li, J.-T., Murphy, E.J., Nikiel-Wroczyński, B., Piotrowska, J., Rand, R.J., Shimwell, T., Stein, Y., Vargas, C.J., Wang, Q.D., van Weeren, R.J., Wiegert, T.: Mon. Not. R. Astron. Soc. (2021). https://doi.org/10.1093/mnras/stab2804. 2109.12267

Heckman, T.M., Borthakur, S.: Astrophys. J. 822, 9 (2016). https://doi. org/10.3847/0004-637X/822/1/9. 1603.03036

Heckman, T.M., Lehnert, M.D., Strickland, D.K., Armus, L.: Astrophys. J. Suppl. Ser. 129, 493 (2000). https://doi.org/10.1086/ 313421. arXiv:astro-ph/0002526

Heckman, T.M., Alexandroff, R.M., Borthakur, S., Overzier, R., Leitherer, C.: Astrophys. J. 809, 147 (2015). https://doi.org/10.1088/ 0004-637X/809/2/147. 1507.05622

Heesen, V., Beck, R., Krause, M., Dettmar, R.-J.: Astron. Astrophys. 494, 563 (2009). https://doi.org/10.1051/0004-6361:200810543. 0812.0346

Heesen, V., Rau, U., Rupen, M.P., Brinks, E., Hunter, D.A.: Astrophys. J. Lett. 739, 23 (2011). https://doi.org/10.1088/2041-8205/739/1/ L23. 1108.0401

Heesen, V., Brinks, E., Leroy, A.K., Heald, G., Braun, R., Bigiel, F., Beck, R.: Astron. J. 147, 103 (2014). https://doi.org/10.1088/ 0004-6256/147/5/103. 1402.1711

Heesen, V., Dettmar, R.-J., Krause, M., Beck, R., Stein, Y.: Mon. Not. R. Astron. Soc. 458, 332 (2016). https://doi.org/10.1093/mnras/ stw360. 1602.04085 
Heesen, V., Rafferty, D.A., Horneffer, A., Beck, R., Basu, A., Westcott, J., Hindson, L., Brinks, E., ChyŻy, K.T., Scaife, A.M.M., Brüggen, M., Heald, G., Fletcher, A., Horellou, C., Tabatabaei, F.S., Paladino, R., Nikiel-Wroczyński, B., Hoeft, M., Dettmar, R.J.: Mon. Not. R. Astron. Soc. 476, 1756 (2018a). https://doi.org/ 10.1093/mnras/sty325

Heesen, V., Krause, M., Beck, R., Adebahr, B., Bomans, D.J., Carretti, E., Dumke, M., Heald, G., Irwin, J., Koribalski, B.S., Mulcahy, D.D., Westmeier, T., Dettmar, R.-J.: Mon. Not. R. Astron. Soc. 476, 158 (2018b). https://doi.org/10.1093/mnras/sty105

Heesen, V., Buie, I.E., Huff, C.J., Perez, L.A., Woolsey, J.G., Rafferty, D.A., Basu, A., Beck, R., Brinks, E., Horellou, C., Scannapieco, E., Brüggen, M., Dettmar, R.-J., Sendlinger, K., NikielWroczyński, B., Chyży, K.T., Best, P.N., Heald, G.H., Paladino, R.: Astron. Astrophys. 622, 8 (2019a). https://doi.org/10.1051/ 0004-6361/201833905. 1811.07968

Heesen, V., Whitler, L., Schmidt, P., Miskolczi, A., Sridhar, S.S., Horellou, C., Beck, R., Gürkan, G., Scannapieco, E., Brüggen, M., Heald, G.H., Krause, M., Paladino, R., Nikiel-Wroczyński, B., Wilber, A., Dettmar, R.-J.: Astron. Astrophys. 628, 3 (2019b). https://doi.org/10.1051/0004-6361/201936046. 1907.07076

Ho, I.-T., Medling, A.M., Bland-Hawthorn, J., Groves, B., Kewley, L.J., Kobayashi, C., Dopita, M.A., Leslie, S.K., Sharp, R., Allen, J.T., Bourne, N., Bryant, J.J., Cortese, L., Croom, S.M., Dunne, L., Fogarty, L.M.R., Goodwin, M., Green, A.W., Konstantopoulos, I.S., Lawrence, J.S., Lorente, N.P.F., Owers, M.S., Richards, S., Sweet, S.M., Tescari, E., Valiante, E.: Mon. Not. R. Astron. Soc. 457(2), 1257 (2016). https://doi.org/10.1093/mnras/stw017. 1601.02022

Hodges-Kluck, E.J., Bregman, J.N.: Astrophys. J. 762, 12 (2013). https://doi.org/10.1088/0004-637X/762/1/12. 1211.1669

Hopkins, P.F., Chan, T.K., Garrison-Kimmel, S., Ji, S., Su, K.-Y., Hummels, C.B., Kereš, D., Quataert, E., Faucher-Giguère, C.-A.: Mon. Not. R. Astron. Soc. 492(3), 3465 (2020). https://doi.org/10.1093/ mnras/stz3321. 1905.04321

Ipavich, F.M.: Astrophys. J. 196, 107 (1975)

Irwin, J., Beck, R., Benjamin, R.A., Dettmar, R.-J., English, J., Heald, G., Henriksen, R.N., Johnson, M., Krause, M., Li, J.-T., Miskolczi, A., Mora, S.C., Murphy, E.J., Oosterloo, T., Porter, T.A., Rand, R.J., Saikia, D.J., Schmidt, P., Strong, A.W., Walterbos, R., Wang, Q.D., Wiegert, T.: Astron. J. 144, 43 (2012). https://doi. org/10.1088/0004-6256/144/2/43. 1205.5694

Israel, F.P., Mahoney, M.J.: Astrophys. J. 352, 30 (1990). https://doi. org/10.1086/168513

Jacob, S., Pakmor, R., Simpson, C.M., Springel, V., Pfrommer, C.: Mon. Not. R. Astron. Soc. 475, 570 (2018). https://doi.org/10. 1093/mnras/stx3221. 1712.04947

Krause, M., Wielebinski, R., Dumke, M.: Astron. Astrophys. 448, 133 (2006). https://doi.org/10.1051/0004-6361:20053789. arXiv: astro-ph/0510796

Krause, M., Irwin, J., Wiegert, T., Miskolczi, A., Damas-Segovia, A., Beck, R., Li, J.-T., Heald, G., Müller, P., Stein, Y., Rand, R.J., Heesen, V., Walterbos, R.A.M., Dettmar, R.-J., Vargas, C.J., English, J., Murphy, E.J.: Astron. Astrophys. 611, 72 (2018). https:// doi.org/10.1051/0004-6361/201731991

Krause, M.G.H., Rodgers-Lee, D., Dale, J.E., Diehl, R., Kobayashi, C.: Mon. Not. R. Astron. Soc. 501(1), 210 (2021). https://doi.org/10. 1093/mnras/staa3612. 2011.08615

Lacki, B.C., Thompson, T.A., Quataert, E., Loeb, A., Waxman, E.: Astrophys. J. 734(2), 107 (2011). https://doi.org/10.1088/0004637X/734/2/107. 1003.3257

Lamers, H.J.G.L.M., Cassinelli, J.P.: Introduction to Stellar Winds, (1999)

Lisenfeld, U., Völk, H.J.: Astron. Astrophys. 354, 423 (2000). astro$\mathrm{ph} / 9912232$

Longair, M.S.: High Energy Astrophysics. Cambridge University Press, Cambridge (2011)
López-Cobá, C., Sánchez, S.F., Bland-Hawthorn, J., Moiseev, A.V., Cruz- González, I., García-Benito, R., Barrera-Ballesteros, J.K., Galbany, L.: Mon. Not. R. Astron. Soc. 482, 4032 (2019). https:// doi.org/10.1093/mnras/sty2960. 1811.01253

Mao, S.A., Ostriker, E.C.: Astrophys. J. 854, 89 (2018). https://doi.org/ 10.3847/1538-4357/aaa88e. 1801.06544

Martin, C.L.: Astrophys. J. 621, 227 (2005). https://doi.org/10.1086/ 427277. astro-ph/0410247

Marvil, J., Owen, F., Eilek, J.: Astron. J. 149, 32 (2015). https://doi. org/10.1088/0004-6256/149/1/32. 1408.6296

Miskolczi, A., Heesen, V., Horellou, C., Bomans, D.-J., Beck, R., Heald, G., Dettmar, R.-J., Blex, S., Nikiel-Wroczyński, B., Chyży, K.T., Stein, Y., Irwin, J.A., Shimwell, T.W., Wang, Q.D.: ArXiv e-prints, 1811 (2018). 1811.04015

Mora-Partiarroyo, S.C., Krause, M., Basu, A., Beck, R., Wiegert, T., Irwin, J., Henriksen, R., Stein, Y., Vargas, C.J., Heesen, V., Walterbos, R.A.M., Rand, R.J., Heald, G., Li, J., Kamieneski, P., English, J.: Astron. Astrophys. 632, 10 (2019a). https://doi.org/10. 1051/0004-6361/201834571. 1910.07588

Mora-Partiarroyo, S.C., Krause, M., Basu, A., Beck, R., Wiegert, T., Irwin, J., Henriksen, R., Stein, Y., Vargas, C.J., Heesen, V., Walterbos, R.A.M., Rand, R.J., Heald, G., Li, J., Kamieneski, P., English, J.: Astron. Astrophys. 632, 11 (2019b). https://doi.org/10. 1051/0004-6361/201935961. 1910.07590

Mulcahy, D.D., Horneffer, A., Beck, R., Heald, G., Fletcher, A., Scaife, A., Adebahr, B., Anderson, J.M., Bonafede, A., Brüggen, M., Brunetti, G., Chyży, K.T., Conway, J., Dettmar, R.-J., Enßlin, T., Haverkorn, M., Horellou, C., Iacobelli, M., Israel, F.P., Junklewitz, H., Jurusik, W., Köhler, J., Kuniyoshi, M., Orrú, E., Paladino, R., Pizzo, R., Reich, W., Röttgering, H.J.A.: Astron. Astrophys. 568, 74 (2014). https://doi.org/10.1051/0004-6361/ 201424187. 1407.1312

Mulcahy, D.D., Fletcher, A., Beck, R., Mitra, D., Scaife, A.M.M.: Astron. Astrophys. 592, 123 (2016). https://doi.org/10.1051/00046361/201628446. 1605.01406

Mulcahy, D.D., Horneffer, A., Beck, R., Krause, M., Schmidt, P., Basu, A., Chyży, K.T., Dettmar, R.-J., Haverkorn, M., Heald, G., Heesen, V., Horellou, C., Iacobelli, M., Nikiel- Wroczyński, B., Paladino, R., Scaife, A.M.M., Sridhar, S.S., Strom, R.G., Tabatabaei, F.S., Cantwell, T., Carey, S.H., Grainge, K., Hickish, J., Perrot, Y., Razavi-Ghods, N., Scott, P., Titterington, D.: Astron. Astrophys. 615, 98 (2018). https://doi.org/10.1051/00046361/201832837

Murphy, E.J., Helou, G., Kenney, J.D.P., Armus, L., Braun, R.: Astrophys. J. 678, 828 (2008). https://doi.org/10.1086/587123. 0801. 4768

Murphy, E.J., Condon, J.J., Schinnerer, E., Kennicutt, R.C., Calzetti, D., Armus, L., Helou, G., Turner, J.L., Aniano, G., Beirão, P., Bolatto, A.D., Brandl, B.R., Croxall, K.V., Dale, D.A., Donovan Meyer, J.L., Draine, B.T., Engelbracht, C., Hunt, L.K., Hao, C.N., Koda, J., Roussel, H., Skibba, R., Smith, J.-D.T.: Astrophys. J. 737(2), 67 (2011). https://doi.org/10.1088/0004-637X/737/2/67. 1105.4877

Murray, N., Quataert, E., Thompson, T.A.: Astrophys. J. 618, 569 (2005). https://doi.org/10.1086/426067. astro-ph/0406070

Norman, C.A., Ikeuchi, S.: Astrophys. J. 345, 372 (1989). https://doi. org/10.1086/167912

Orlando, E., Strong, A.: Mon. Not. R. Astron. Soc. 436(3), 2127 (2013). https://doi.org/10.1093/mnras/stt1718. 1309.2947

Pakmor, R., Pfrommer, C., Simpson, C.M., Springel, V.: Astrophys. J. Lett. 824, 30 (2016a). https://doi.org/10.3847/2041-8205/824/ 2/L30. 1605.00643

Pakmor, R., Pfrommer, C., Simpson, C.M., Springel, V.: Astrophys. J. Lett. 824, 30 (2016b). https://doi.org/10.3847/2041-8205/824/2/ L30. 1605.00643

Pakmor, R., Gómez, F.A., Grand, R.J.J., Marinacci, F., Simpson, C.M., Springel, V., Campbell, D.J.R., Frenk, C.S., Guillet, T., Pfrommer, 
C., White, S.D.M.: Mon. Not. R. Astron. Soc. 469, 3185 (2017). https://doi.org/10.1093/mnras/stx1074. 1701.07028

Pohl, M., Schlickeiser, R., Hummel, E.: Astron. Astrophys. 250, 302 (1991)

Ptuskin, V.S., Voelk, H.J., Zirakashvili, V.N., Breitschwerdt, D.: Astron. Astrophys. 321, 434 (1997)

Rau, U., Cornwell, T.J.: Astron. Astrophys. 532, 71 (2011). https://doi. org/10.1051/0004-6361/201117104. 1106.2745

Recchia, S., Blasi, P., Morlino, G.: Mon. Not. R. Astron. Soc. 462, 4227 (2016). https://doi.org/10.1093/mnras/stw1966. 1603.06746

Rieger, F.M., de Oña-Wilhelmi, E., Aharonian, F.A.: Frontiers of Physics 8, 714 (2013). https://doi.org/10.1007/s11467-0130344-6. 1302.5603

Rossa, J., Dettmar, R.-J.: Astron. Astrophys. 406, 493 (2003a). https:// doi.org/10.1051/0004-6361:20030615. astro-ph/0304452

Rossa, J., Dettmar, R.-J.: Astron. Astrophys. 406, 505 (2003b). https:// doi.org/10.1051/0004-6361:20030698. astro-ph/0305472

Rupke, D.S., Veilleux, S., Sanders, D.B.: Astrophys. J. Suppl. Ser. 160, 115 (2005). https://doi.org/10.1086/432889. astro-ph/0506611

Ruszkowski, M., Yang, H.-Y.K., Zweibel, E.: Astrophys. J. 834(2), 208 (2017). https://doi.org/10.3847/1538-4357/834/2/208. 1602. 04856

Salem, M., Bryan, G.L.: Mon. Not. R. Astron. Soc. 437, 3312 (2014). https://doi.org/10.1093/mnras/stt2121

Samui, S., Subramanian, K., Srianand, R.: Mon. Not. R. Astron. Soc. 402, 2778 (2010). https://doi.org/10.1111/j.1365-2966.2009. 16099.x. 0909.3854

Schmidt, P., Krause, M., Heesen, V., Basu, A., Beck, R., Wiegert, T., Irwin, J.A., Heald, G., Rand, R.J., Li, J.-T., Murphy, E.J.: Astron. Astrophys. 632, 12 (2019). https://doi.org/10.1051/00046361/201834995. 1907.03789

Shalchi, A., Škoda, T., Tautz, R.C., Schlickeiser, R.: Phys. Rev. D 80(2), 023012 (2009). https://doi.org/10.1103/PhysRevD. 80.023012

Sharma, P., Roy, A., Nath, B.B., Shchekinov, Y.: Mon. Not. R. Astron. Soc. 443(4), 3463 (2014). https://doi.org/10.1093/mnras/stu1307. 1402.6695

Singal, J., Kogut, A., Jones, E., Dunlap, H.: Astrophys. J. 799, 10 (2015). https://doi.org/10.1088/2041-8205/799/1/L10

Socrates, A., Davis, S.W., Ramirez-Ruiz, E.: Astrophys. J. 687(1), 202 (2008). https://doi.org/10.1086/590046. astro-ph/0609796

Soida, M., Krause, M., Dettmar, R.-J., Urbanik, M.: Astron. Astrophys. 531, 127 (2011). https://doi.org/10.1051/0004-6361/200810763. 1105.5259

Stein, Y., Dettmar, R.-J., Irwin, J., Beck, R., Weżgowiec, M., Miskolczi, A., Krause, M., Heesen, V., Wiegert, T., Heald, G., Walterbos, R.A.M., Li, J.-T., Soida, M.: Astron. Astrophys. 623, 33 (2019a). https://doi.org/10.1051/0004-6361/201834515. 1901.08090

Stein, Y., Dettmar, R.-J., Weżgowiec, M., Irwin, J., Beck, R., Wiegert, T., Krause, M., Li, J.-T., Heesen, V., Miskolczi, A., MacDonald, S., English, J.: Astron. Astrophys. 632, 13 (2019b). https://doi. org/10.1051/0004-6361/201935558. 1906.10650

Stein, Y., Dettmar, R.-J., Beck, R., Irwin, J., Wiegert, T., Miskolczi, A., Wang, Q.D., English, J., Henriksen, R., Radica, M., Li, J.T.: Astron. Astrophys. 639, 111 (2020). https://doi.org/10.1051/ 0004-6361/202037675. 2007.03002

Steinwandel, U.P., Dolag, K., Lesch, H., Moster, B.P., Burkert, A., Prieto, A.: Mon. Not. R. Astron. Soc. 494(3), 4393 (2020). https:// doi.org/10.1093/mnras/staa817. 1907.11727

Strickland, D.K., Heckman, T.M., Colbert, E.J.M., Hoopes, C.G., Weaver, K.A.: Astrophys. J. Suppl. Ser. 151, 193 (2004). https:// doi.org/10.1086/382214. astro-ph/0306592

Strong, A.W., Moskalenko, I.V., Ptuskin, V.S.: Annu. Rev. Nucl. Part. Sci. 57, 285 (2007). https://doi.org/10.1146/annurev.nucl.57. 090506.123011. astro-ph/0701517
Tabatabaei, F.S., Schinnerer, E., Murphy, E.J., Beck, R., Groves, B., Meidt, S., Krause, M., Rix, H.-W., Sandstrom, K., Crocker, A.F., Galametz, M., Helou, G., Wilson, C.D., Kennicutt, R., Calzetti, D., Draine, B., Aniano, G., Dale, D., Dumas, G., Engelbracht, C.W., Gordon, K.D., Hinz, J., Kreckel, K., Montiel, E., Roussel, H.: Astron. Astrophys. 552, 19 (2013). https://doi.org/10.1051/ 0004-6361/201220249. 1301.6884

Tabatabaei, F.S., Schinnerer, E., Krause, M., Dumas, G., Meidt, S., Damas-Segovia, A., Beck, R., Murphy, E.J., Mulcahy, D.D., Groves, B., Bolatto, A., Dale, D., Galametz, M., Sandstrom, K., Boquien, M., Calzetti, D., Kennicutt, R.C., Hunt, L.K., De Looze, I., Pellegrini, E.W.: Astrophys. J. 836, 185 (2017). https://doi.org/ 10.3847/1538-4357/836/2/185. 1611.01705

Tüllmann, R., Dettmar, R.-J., Soida, M., Urbanik, M., Rossa, J.: Astron. Astrophys. 364, 36 (2000). arXiv:astro-ph/0010267

Tüllmann, R., Pietsch, W., Rossa, J., Breitschwerdt, D., Dettmar, R.-J.: Astron. Astrophys. 448, 43 (2006). https://doi.org/10.1051/00046361:20052936. astro-ph/0510079

Tumlinson, J., Peeples, M.S., Werk, J.K.: Annu. Rev. Astron. Astrophys. 55(1), 389 (2017). https://doi.org/10.1146/annurev-astro091916-055240. 1709.09180

Uhlig, M., Pfrommer, C., Sharma, M., Nath, B.B., Enßlin, T.A., Springel, V.: Mon. Not. R. Astron. Soc. 423(3), 2374 (2012). https://doi.org/10.1111/j.1365-2966.2012.21045.x. 1203.1038

van Haarlem, M.P., Wise, M.W., Gunst, A.W., Heald, G., McKean, J.P., Hessels, J.W.T., de Bruyn, A.G., Nijboer, R., Swinbank, J., Fallows, R., Brentjens, M., Nelles, A., Beck, R., Falcke, H., Fender, R., Hörandel, J., Koopmans, L.V.E., Mann, G., Miley, G., Röttgering, H., Stappers, B.W., Wijers, R.A.M.J., Zaroubi, S., van den Akker, M., Alexov, A., Anderson, J., Anderson, K., van Ardenne, A., Arts, M., Asgekar, A., Avruch, I.M., Batejat, F., Bähren, L., Bell, M.E., Bell, M.R., van Bemmel, I., Bennema, P., Bentum, M.J., Bernardi, G., Best, P., Bîrzan, L., Bonafede, A., Boonstra, A.-J., Braun, R., Bregman, J., Breitling, F., van de Brink, R.H., Broderick, J., Broekema, P.C., Brouw, W.N., Brüggen, M., Butcher, H.R., van Cappellen, W., Ciardi, B., Coenen, T., Conway, J., Coolen, A., Corstanje, A., Damstra, S., Davies, O., Deller, A.T., Dettmar, R.-J., van Diepen, G., Dijkstra, K., Donker, P., Doorduin, A., Dromer, J., Drost, M., van Duin, A., Eislöffel, J., van Enst, J., Ferrari, C., Frieswijk, W., Gankema, H., Garrett, M.A., de Gasperin, F., Gerbers, M., de Geus, E., Grießmeier, J.-M., Grit, T., Gruppen, P., Hamaker, J.P., Hassall, T., Hoeft, M., Holties, H.A., Horneffer, A., van der Horst, A., van Houwelingen, A., Huijgen, A., Iacobelli, M., Intema, H., Jackson, N., Jelic, V., de Jong, A., Juette, E., Kant, D., Karastergiou, A., Koers, A., Kollen, H., Kondratiev, V.I., Kooistra, E., Koopman, Y., Koster, A., Kuniyoshi, M., Kramer, M., Kuper, G., Lambropoulos, P., Law, C., van Leeuwen, J., Lemaitre, J., Loose, M., Maat, P., Macario, G., Markoff, S., Masters, J., McFadden, R.A., McKay-Bukowski, D., Meijering, H., Meulman, H., Mevius, M., Middelberg, E., Millenaar, R., Miller-Jones, J.C.A., Mohan, R.N., Mol, J.D., Morawietz, J., Morganti, R., Mulcahy, D.D., Mulder, E., Munk, H., Nieuwenhuis, L., van Nieuwpoort, R., Noordam, J.E., Norden, M., Noutsos, A., Offringa, A.R., Olofsson, H., Omar, A., Orrú, E., Overeem, R., Paas, H., PandeyPommier, M., Pandey, V.N., Pizzo, R., Polatidis, A., Rafferty, D., Rawlings, S., Reich, W., de Reijer, J.-P., Reitsma, J., Renting, G.A., Riemers, P., Rol, E., Romein, J.W., Roosjen, J., Ruiter, M., Scaife, A., van der Schaaf, K., Scheers, B., Schellart, P., Schoenmakers, A., Schoonderbeek, G., Serylak, M., Shulevski, A., Sluman, J., Smirnov, O., Sobey, C., Spreeuw, H., Steinmetz, M., Sterks, C.G.M., Stiepel, H.-J., Stuurwold, K., Tagger, M., Tang, Y., Tasse, C., Thomas, I., Thoudam, S., Toribio, M.C., van der Tol, B., Usov, O., van Veelen, M., van der Veen, A.-J., ter Veen, S., Verbiest, J.P.W., Vermeulen, R., Vermaas, N., Vocks, C., Vogt, C., de Vos, M., van der Wal, E., van Weeren, R., Weggemans, H., Weltevrede, P., White, S., Wijnholds, S.J., Wilhelmsson, 
T., Wucknitz, O., Yatawatta, S., Zarka, P., Zensus, A., van Zwieten, J.: Astron. Astrophys. 556, 2 (2013). https://doi.org/10.1051/ 0004-6361/201220873. 1305.3550

Vasiliev, E.O., Shchekinov, Y.A., Nath, B.B.: arXiv e-prints, 1901 (2019). 1901.00821

Veilleux, S., Maiolino, R., Bolatto, A.D., Aalto, S.: Astron. Astrophys. Rev. 28(1), 2 (2020). https://doi.org/10.1007/s00159-019-0121-9. 2002.07765

Veilleux, S., Meléndez, M., Stone, M., Cecil, G., Hodges-Kluck, E., Bland-Hawthorn, J., Bregman, J., Heitsch, F., Martin, C.L., Mueller, T., Rupke, D.S.N., Sturm, E., Tanner, R., Engelbracht, C.: Mon. Not. R. Astron. Soc. (2021). https://doi.org/10.1093/ mnras/stab2881. 2110.01766

Vijayan, A., Nath, B.B., Sharma, P., Shchekinov, Y.: Mon. Not. R. Astron. Soc. 492(2), 2924 (2020). https://doi.org/10.1093/mnras/ stz3568. 1911.07870

Vogelsberger, M., Marinacci, F., Torrey, P., Puchwein, E.: Nature Reviews Physics 2(1), 42 (2020). https://doi.org/10.1038/s42254019-0127-2. 1909.07976

Vollmer, B., Soida, M., Beck, R., Powalka, M.: Astron. Astrophys. 633, 144 (2020). https://doi.org/10.1051/0004-6361/201935923. 1911. 02253

Westmoquette, M.S., Smith, L.J., Gallagher, J.S. III: Mon. Not. R. Astron. Soc. 414, 3719 (2011). https://doi.org/10.1111/j.1365-2966. 2011.18675.x. 1103.1775

Wiegert, T., Irwin, J., Miskolczi, A., Schmidt, P., Mora, S.C., DamasSegovia, A., Stein, Y., English, J., Rand, R.J., Santistevan, I., Walterbos, R., Krause, M., Beck, R., Dettmar, R.-J., Kepley, A., Wezgowiec, M., Wang, Q.D., Heald, G., Li, J., MacGregor,
S., Johnson, M., Strong, A.W., DeSouza, A., Porter, T.A.: Astron. J. 150, 81 (2015). https://doi.org/10.1088/0004-6256/150/ 3/81. 1508.05153

Wiener, J., Pfrommer, C., Oh, S.P.: Mon. Not. R. Astron. Soc. 467, 906 (2017). https://doi.org/10.1093/mnras/stx127. 1608.02585

Yoast-Hull, T.M., Everett, J.E., Gallagher, J.S. III, Zweibel, E.G.: Astrophys. J. 768, 53 (2013). https://doi.org/10.1088/0004-637X/ 768/1/53. 1303.4305

Yoast-Hull, T.M., Gallagher, J.S. III, Zweibel, E.G., Everett, J.E.: Astrophys. J. 780(2), 137 (2014). https://doi.org/10.1088/0004637X/780/2/137. 1311.5586

Yoon, J.H., Martin, C.L., Veilleux, S., Meléndez, M., Mueller, T., Gordon, K.D., Cecil, G., Bland-Hawthorn, J., Engelbracht, C.: Mon. Not. R. Astron. Soc. 502(1), 969 (2021). https://doi.org/10.1093/ mnras/staa3583. 2012.08686

Yu, B.P.B., Owen, E.R., Wu, K., Ferreras, I.: Mon. Not. R. Astron. Soc. 492(3), 3179 (2020). https://doi.org/10.1093/mnras/staa021. 2001.04384

Zirakashvili, V.N., Breitschwerdt, D., Ptuskin, V.S., Völk, H.J.: Astron. Astrophys. 311, 113 (1996)

Zschaechner, L.K., Rand, R.J., Walterbos, R.: Astrophys. J. 799, 61 (2015). https://doi.org/10.1088/0004-637X/799/1/61. 1411.2593

Zweibel, E.G.: Physics of Plasmas 20(5), 055501 (2013). https://doi. org/10.1063/1.4807033

Publisher's Note Springer Nature remains neutral with regard to jurisdictional claims in published maps and institutional affiliations. 